MATHEMATICS OF COMPUTATION

Volume 76, Number 258, April 2007, Pages 509-537

S 0025-5718(06)01910-7

Article electronically published on October 17, 2006

\title{
ON THE FINITE ELEMENT METHOD FOR ELLIPTIC PROBLEMS WITH DEGENERATE AND SINGULAR COEFFICIENTS
}

\author{
DANIEL ARROYO, ALEXEI BESPALOV, AND NORBERT HEUER
}

\begin{abstract}
We consider Dirichlet boundary value problems for second order elliptic equations over polygonal domains. The coefficients of the equations under consideration degenerate at an inner point of the domain, or behave singularly in the neighborhood of that point. This behavior may cause singularities in the solution. The solvability of the problems is proved in weighted Sobolev spaces, and their approximation by finite elements is studied. This study includes regularity results, graded meshes, and inverse estimates. Applications of the theory to some problems appearing in quantum mechanics are given. Numerical results are provided which illustrate the theory and confirm the predicted rates of convergence of the finite element approximations for quasi-uniform meshes.
\end{abstract}

\section{INTRODUCTION}

Let $\Omega \subset \mathbb{R}^{2}=\left\{x=\left(x_{1}, x_{2}\right) \mid x_{i} \in \mathbb{R}, i=1,2\right\}$ be a convex polygonal domain, and let $\bar{\Omega}=\Omega \cup \partial \Omega$. Assume that the origin $O=(0,0)$ is an interior point of $\Omega$, and denote by $r=r(x)=\left(x_{1}^{2}+x_{2}^{2}\right)^{1 / 2}$ the distance of $x \in \bar{\Omega}$ to the origin $O$.

We shall consider the following problem:

$$
\begin{aligned}
-\operatorname{div}\left(a r^{2 \beta} \nabla u\right)+b r^{2 \alpha} u & =f \text { in } \Omega, \\
u & =0 \text { on } \partial \Omega,
\end{aligned}
$$

where $\alpha>-1$ and $\beta>-1$ are real, and $a$ and $b$ are measurable functions satisfying the inequalities

$$
0<\underline{a} \leq a(x) \leq \bar{a}, \quad 0<\underline{b} \leq b(x) \leq \bar{b} \quad \text { almost everywhere in } \bar{\Omega} .
$$

Observe that the coefficients of the equation in (1.1) degenerate at the origin or have singular behavior in the neighborhood of this point (unless $\alpha=\beta=0$ ). Later we shall also study problems of this type in the nondivergence form. Such problems with degenerate or singular coefficients appear, e.g., in quantum mechanics when modeling the motion of particles in potential fields.

Received by the editor November 17, 2003 and, in revised form, July 21, 2005.

2000 Mathematics Subject Classification. Primary 65N30, 65N15.

Key words and phrases. Finite element method, problems with singularities, Coulomb field.

The first author was supported by Fondecyt project 1010220, Chile.

The second author was supported by the Russian Foundation for Basic Research project no. 01-01-00375 and by the FONDAP Program in Applied Mathematics, Chile.

The third author was supported by the FONDAP Program in Applied Mathematics and Fondecyt projects 1010220, 1040615, Chile. 
Depending on the coefficients $a, b$ and the parameters $\alpha$ and $\beta$, the solution of (1.1) can be infinite at the origin (the case of a strong singularity, $u \notin H^{1}(\Omega)$ ). Regular or weakly singular solutions $\left(u \in H^{1}(\Omega)\right.$ but $\left.u \notin H^{2}(\Omega)\right)$ may also appear. Due to the nonregular coefficients and in the presence of strong singularities the standard formulation of problem (1.1) in the Sobolev space $H^{1}(\Omega)$ is not appropriate. In this paper we present a framework which deals with all these cases.

At present there exist several approaches to treat boundary value problems with degenerate (singular) data. These approaches usually depend on the character of the arising singularities. In the one-dimensional case, such problems and their finite element approximations were investigated in [13, 9]. The finite element approximation for the special case of a right-hand side with the Dirac $\delta$-distribution was analyzed in [1, 19, 8, 7. A class of problems where the coefficients and the right-hand side function have singularities on the boundary is treated in [15, 16]. The problem similar to (1.1) with degenerate (singular) coefficients and a strongly singular solution at a boundary point was considered in [17, 18, 5, 4]. In these papers the case $\alpha=\beta-1$ was studied. For that case, using a special variational formulation in weighted Sobolev-Kondrat'ev spaces, the unique solvability of the problem was proved and various finite element approximations (the $h-, p-$, and the $h-p$ versions) were investigated.

The aim of this paper is to present a weak formulation of problem (1.1) which ensures unique solvability, to construct a finite element scheme, and to analyze its convergence. Depending on the specific problem (behavior of the coefficients) the solution of (1.1) behaves singularly. For such cases we prove that the use of graded meshes ensures an optimal rate of convergence. We also present regularity results and prove for some cases the optimality or quasi-optimality of the a priori error estimates. For model problems we report on several numerical experiments which confirm our theoretical results for quasi-uniform meshes.

The paper is organized as follows. In $\$ 2$ we introduce the basic notation and formulate the main results. In $\$ 2.1$ we present a variational formulation of problem (1.1), establish its unique solvability (Theorem 2.1), construct the finite element scheme, and state a priori error estimates for this approximation on quasi-uniform and graded meshes (Theorem 2.2). In this subsection we also study the regularity of the solution to problem (1.1) (Theorem 2.3) and prove the quasi-optimality of the a priori error estimate on quasi-uniform meshes (Theorem 2.4). Theorem 2.5 proves an inverse estimate in weighted Besov spaces which also serves to prove optimality of a priori error estimates. In $\$ 2.2$ we demonstrate how the theory applies to two kinds of problems in nondivergence form appearing in quantum mechanics. In order to prove the main results, in $\$ 3$ we first collect some technical lemmas regarding the properties of weighted Sobolev spaces and give the proofs of the theorems. Finally, $\$ 4$ presents some numerical results illustrating and confirming the theory.

\section{MAIN RESUlts}

Before stating the main results let us introduce the spaces and norms which will be used.

Throughout the paper, we shall assume that $\bar{\Omega} \subset B$, where $B$ is the unit disk with center at the origin. For any real $\eta$ we shall denote by $L_{2, \eta}(\Omega)$ the space of functions which are square integrable with the weight $r^{2 \eta}$ on $\Omega$, furnished with the 
following inner product and norm:

$$
(u, v)_{L_{2, \eta}(\Omega)}=\int_{\Omega} r^{2 \eta}(x) u(x) v(x) d x, \quad\|u\|_{L_{2, \eta}(\Omega)}=(u, u)_{L_{2, \eta}(\Omega)}^{1 / 2} .
$$

For $\eta=0$, we have the classical Lebesgue space of index 2 on $\Omega$ : $L_{2,0}(\Omega)=L_{2}(\Omega)$.

Now, for any integer $k \geq 1$, we introduce the space $H_{\eta_{0}, \eta_{1}, \ldots, \eta_{k}}^{k}(\Omega)$ with real $\eta_{l}>-1(l=0,1, \ldots, k)$ as the completion of the set of all infinitely differentiable functions on $\Omega$ under the norm defined by

$$
\|u\|_{H_{\eta_{0}, \eta_{1}, \ldots, \eta_{k}}^{k}(\Omega)}^{2}=\sum_{l=0}^{k}\left\|r^{\eta_{l}} \mid D^{l} u\right\|_{L_{2}(\Omega)}^{2} .
$$

Here, $\left|D^{l} u\right|^{2}(x)=\sum_{|\lambda|=l}\left|D^{\lambda} u(x)\right|^{2}=\sum_{|\lambda|=l}\left|\frac{\partial^{|\lambda|} u(x)}{\partial x_{1}^{\lambda_{1}} \partial x_{2}^{\lambda_{2}}}\right|^{2}, \lambda=\left(\lambda_{1}, \lambda_{2}\right), \lambda_{i} \geq 0$, are integers $(i=1,2)$, and $|\lambda|=\lambda_{1}+\lambda_{2}$. If $\eta_{0}=\eta_{1}=\cdots=\eta_{k}=0$, then we obviously have the standard Sobolev space $H^{k}(\Omega)$ with the norm $\|\cdot\|_{H^{k}(\Omega)}$ and semi-norm $|u|_{H^{k}(\Omega)}=\left\|D^{k} u\right\|_{L_{2}(\Omega)}$. If $k=2$, and $\eta_{0}=\eta_{1}=0, \eta_{2}=\eta \in[0,1)$, then according to [11] we shall use the notation $H_{0,0, \eta}^{2}(\Omega)=H_{\eta}^{2,2}(\Omega)$. Quoting [10, Lemma 8.4.1.2] observe that

$$
H_{\eta}^{2,2}(\Omega) \subset C^{0}(\bar{\Omega}),
$$

where $C^{0}(\bar{\Omega})$ is the space of continuous functions on $\bar{\Omega}$, furnished with the norm

$$
\|u\|_{C^{0}(\bar{\Omega})}=\max _{x \in \bar{\Omega}}|u(x)| .
$$

By $\stackrel{\circ}{H}_{\eta_{0}, \eta_{1}}^{1}(\Omega)$ we denote the completion of the set of all infinitely differentiable functions with compact support in $\Omega$, under the norm $\|\cdot\|_{H_{\eta_{0}, \eta_{1}}^{1}(\Omega)}$.

We shall also use the Besov spaces defined by interpolation between the above weighted spaces. We use the theory of interpolation spaces developed, e.g., in [3]. For $u \in \stackrel{\circ}{H}_{\eta_{0}, \eta_{1}}^{1}(\Omega)$ we set

$$
K(t, u)=\inf _{u=v+w}\left(\|v\|_{H_{\eta_{0}, \eta_{1}}^{1}(\Omega)}+t\|w\|_{H_{\eta_{0}, \eta_{1}, \eta_{2}}^{2}(\Omega)}\right) .
$$

Here, $v$ and $w$ must satisfy the homogeneous boundary conditions, i.e., $v \in \stackrel{\circ}{H}_{\eta_{0}, \eta_{1}}^{1}(\Omega)$ and $w \in H_{\eta_{0}, \eta_{1}, \eta_{2}}^{2}(\Omega) \cap \stackrel{\circ}{H_{\eta_{0}, \eta_{1}}^{1}}(\Omega)$. Then for $\theta \in(0,1)$ the Besov space $X_{\theta}=$ $X\left(1, \eta_{0}, \eta_{1}, 2, \eta_{2}, \theta, \infty\right)$ is defined as the set of all functions $u \in \stackrel{\circ}{H}_{\eta_{0}, \eta_{1}}^{1}(\Omega)$ satisfying

$$
\|u\|_{X_{\theta}}=\sup _{t>0}\left\{t^{-\theta} K(t, u)\right\}<\infty .
$$

2.1. The model problem in divergence form. We begin by investigating the model problem (1.1) in divergence form. In $\$ 2.2$ below, we demonstrate how the theory applies to some problems which are not given in divergence form.

We shall assume that the right-hand side function $f$ in (1.1) is in the space $L_{2, \mu}(\Omega), \mu \in \mathbb{R}$. We define a bilinear form associated with the elliptic operator in (1.1) by

$$
A(u, v)=\int_{\Omega}\left(a r^{2 \beta} \nabla u \nabla v+b r^{2 \alpha} u v\right) d x
$$


and the linear functional

$$
F(v)=\int_{\Omega} f v d x .
$$

Then problem (1.1) can be posed in the following weak form:

$$
\text { find } u \in \stackrel{\circ}{H}_{\alpha, \beta}^{1}(\Omega) \text { satisfying } A(u, v)=F(v) \text { for all } v \in \stackrel{\circ}{H}_{\alpha, \beta}^{1}(\Omega) .
$$

Theorem 2.1. Let

$$
\mu \leq \begin{cases}-\alpha, & \text { if }-1<\beta<0, \\ \max \{-\alpha, 1-\beta\}, & \text { if } \beta>0,\end{cases}
$$

and let

$$
\mu<1, \quad \text { if } \beta=0 .
$$

Then, for given $f \in L_{2, \mu}(\Omega)$, there exists a unique weak solution $u \in \stackrel{\circ}{H}_{\alpha, \beta}^{1}(\Omega)$ to problem (1.1), and there holds (for a constant $C$ independent of $f$ )

$$
\|u\|_{H_{\alpha, \beta}^{1}(\Omega)} \leq C\|f\|_{L_{2, \mu}(\Omega)} .
$$

For the approximate solution of problem (1.1) we apply the finite element method (FEM), with quasi-uniform or graded meshes. The scheme of the FEM is constructed on the basis of the weak formulation (2.2).

Let $\Delta=\left\{\Omega_{i} \mid i=1, \ldots, m\right\}(m \geq 3)$ be a triangulation of $\Omega$. We require that the singularity point $O$ is an interior node of the mesh. The intersection of any triangles $\bar{\Omega}_{i}, \bar{\Omega}_{k} \in \Delta(i \neq k)$ is a common vertex, an entire side, or empty. For each triangle $\Omega_{i} \in \Delta$ we shall denote by $h_{i}$ (respectively, $\underline{h}_{i}$ ) the maximal (respectively, the minimal) length of the sides of $\Omega_{i}$, and by $d_{i}$ the distance between the origin $O$ and $\Omega_{i}$.

Let $T$ be the standard triangle $T=\left\{\left(\xi_{1}, \xi_{2}\right) \mid 0<\xi_{1}<1,0<\xi_{2}<\xi_{1}\right\}$ in the plane $O^{\prime} \xi_{1} \xi_{2}$. Then, for each $\Omega_{i} \in \Delta$ there exists a linear transformation $M_{i}$ mapping $T$ onto $\bar{\Omega}_{i}$, and the Jacobian $J_{i}$ of the transformation $M_{i}$ satisfies

$$
\sigma \underline{h}_{i}^{2} \leq\left|J_{i}\right| \leq h_{i}^{2},
$$

where $\sigma>0$ is independent of $i$ (it is known that $\left|J_{i}\right| \geq \underline{h}_{i}^{2} \sin \theta_{i}$, where $\theta_{i}$ denotes the minimal angle of $\Omega_{i}$; therefore the constant $\sigma$ in (2.4) controls the shape regularity of the triangulation $\Delta$, and $0<\sigma<1$ ).

Following 2] we introduce graded meshes. For a mesh parameter $h \in(0,1)$ and a grading parameter $\kappa \in[0,1)$ the triangulation $\Delta(h, \kappa, \sigma)=\left\{\Omega_{i} \mid i=1, \ldots, m\right\}$ is called a graded mesh if:

i) for any element $\bar{\Omega}_{i} \not \supset O$ there holds $\sigma h r^{\kappa}(x) \leq h_{i} \leq \sigma^{-1} h r^{\kappa}(x), x \in \bar{\Omega}_{i}$;

ii) for any element $\bar{\Omega}_{i} \ni O$ there holds

$$
\sigma h \sup _{x \in \Omega_{i}} r^{\kappa}(x) \leq h_{i} \leq \sigma^{-1} h \sup _{x \in \Omega_{i}} r^{\kappa}(x) .
$$

Of course, selecting $\kappa=0$ one obtains quasi-uniform meshes.

It has been proved in [2, Lemma 4.1] that the number $N$ of vertices in $\Delta(h, \kappa, \sigma)$ is bounded as $N \leq C h^{-2}$ with $C>0$ independent of $h$ but depending in general on $\Omega, \kappa$, and $\sigma$. Therefore one can estimate the error of approximation on the graded mesh in terms of $h$. 
For the mesh $\Delta=\Delta(h, \kappa, \sigma)$ (or sequence of meshes for a sequence of mesh parameters $h \rightarrow 0$, fixed $\kappa$, and $\sigma$ bounded from below by a positive number) we define the finite element space $S_{h}=S_{h}(\kappa, \sigma)$ in the usual way as

$$
S_{h}(\kappa, \sigma)=\left\{v \in C^{0}(\Omega) \mid v=0 \text { on } \partial \Omega, \quad\left(\left.v\right|_{\Omega_{i}} \circ M_{i}\right) \text { is a linear function on } T\right\},
$$

and consider the following discrete problem:

$$
\text { find } u_{h} \in S_{h} \text { such that } A\left(u_{h}, v\right)=F(v) \text { for all } v \in S_{h} \text {. }
$$

It is obvious that $S_{h} \subset \stackrel{\circ}{H}_{\alpha, \beta}^{1}(\Omega)$ for any $\alpha>-1$ and $\beta>-1$. Therefore the existence and uniqueness of the approximate solution $u_{h} \in S_{h}$ follow by the Lax-Milgram lemma from the properties of $A(u, v)$ and $F(v)$ (see the proof of Theorem 2.11). In addition, using the same arguments as in the proof of Cea's lemma (see [6]), we obtain

$$
\left\|u-u_{h}\right\|_{H_{\alpha, \beta}^{1}(\Omega)} \leq \frac{\max \{\bar{a}, \bar{b}\}}{\min \{\underline{a}, \underline{b}\}} \inf _{v \in S_{h}}\|u-v\|_{H_{\alpha, \beta}^{1}(\Omega)} .
$$

In the following, if not specified otherwise, $C$ denotes a generic positive constant which is independent of the mesh parameter $h$ but may depend on $\sigma$ in (2.4) and the mesh grading parameter $\kappa$.

The following result states the convergence of the finite element scheme. In particular, under the assumed regularity, the optimal rate of linear convergence is always achievable by appropriate mesh grading.

Theorem 2.2. Let $\alpha>-1, \beta>-1$, and $\gamma=\min \{\alpha+1, \beta\}+\varepsilon$ for some $\varepsilon \in[0,1)$. Let $u_{h} \in S_{h}(\kappa, \sigma)$ be the finite element approximation of the weak solution $u$ to problem (1.1). Assume that $u \in \stackrel{\circ}{H}_{\alpha, \beta}^{1}(\Omega) \cap H_{\alpha, \beta, \gamma}^{2}(\Omega)$. Then there holds

$$
\left\|u-u_{h}\right\|_{H_{\alpha, \beta}^{1}(\Omega)} \leq C \begin{cases}h\|u\|_{H_{\alpha, \beta, \gamma}^{2}(\Omega)} & \text { if } \varepsilon \leq \kappa<1 \\ h^{\frac{1-\varepsilon}{1-\kappa}}\|u\|_{H_{\alpha, \beta, \gamma}^{2}}(\Omega) & \text { if } 0 \leq \kappa<\varepsilon .\end{cases}
$$

Interpolating between $\stackrel{\circ}{H}_{\alpha, \beta}^{1}(\Omega)$ and $H_{\alpha, \beta, \gamma}^{2}(\Omega)$ we obtain the following a priori error estimate for less regular functions.

Corollary 2.1. Let $\alpha>-1, \beta>-1$, and $\gamma=\min \{\alpha+1, \beta\}+\varepsilon$ for some $\varepsilon \in[0,1)$. Let $u_{h} \in S_{h}(\kappa, \sigma)$ be the finite element approximation of the weak solution $u$ to problem (1.1). If $u \in X_{\theta}=X(1, \alpha, \beta, 2, \gamma, \theta, \infty)$ with $\theta \in(0,1)$, then

$$
\left\|u-u_{h}\right\|_{H_{\alpha, \beta}^{1}(\Omega)} \leq C \begin{cases}h^{\theta}\|u\|_{X_{\theta}} & \text { if } \varepsilon \leq \kappa<1, \\ h^{\frac{1-\varepsilon}{1-\kappa} \theta}\|u\|_{X_{\theta}} & \text { if } 0 \leq \kappa<\varepsilon .\end{cases}
$$

Under the condition $\alpha \geq \beta-1$ we prove the regularity of the solution to (1.1) as assumed in Theorem 2.2 .

Theorem 2.3. Let $\alpha>-1, \beta \geq 0$, and $\alpha \geq \beta-1$. Let $u \in \stackrel{\circ}{H}_{\alpha, \beta}^{1}(\Omega)$ be the weak solution to problem (1.1) for given right-hand side $f \in L_{2, \mu}(\Omega)$. Then there exists $\varepsilon \in[0,1)$ such that $u \in H_{\alpha, \beta, \beta+\varepsilon}^{2}(\Omega)$ provided that $\mu \leq-\beta+\varepsilon$. Moreover,

$$
\|u\|_{H_{\alpha, \beta, \beta+\varepsilon}^{2}(\Omega)} \leq C\|f\|_{L_{2, \mu}(\Omega)} .
$$


A remaining question is the optimality of the error estimates (2.7), (2.8). Here, we give a partial answer. For sufficiently graded meshes $(\kappa \geq \varepsilon)$ the linear convergence is optimal since piecewise linear polynomials are used in the finite element scheme. For quasi-uniform meshes we show the quasi-optimality of (2.7) by studying an example (see Theorem 2.4). For $\theta \leq 1 / 2$ and for graded meshes with grading parameter $\kappa=\varepsilon$, and under additional assumptions on the mesh construction, the optimality of (2.8) can be proved by an inverse estimate; see [2, Theorem 6.1]. Such an estimate is given by Theorem 2.5 below.

Let us consider problem (1.1) with right-hand side function $f$ given such that

$$
u=u_{\text {model }}:=r^{\lambda} \chi(r) \text {. }
$$

Here, $\lambda \neq 0, \chi$ is a $C^{\infty}$ cut-off function, $\chi(r)=1$ for $0 \leq r \leq \rho, \chi(r)=0$ for $r \geq 2 \rho$, and $\rho>0$ is sufficiently small. To ensure $u_{\text {model }} \in \stackrel{\circ}{H}_{\alpha, \beta}^{1}(\Omega) \cap H_{\alpha, \beta, \min \{\alpha+1, \beta\}+\varepsilon}^{2}(\Omega)$ for some $\varepsilon \in[0,1)$, we assume $\lambda>1-\min \{\alpha+1, \beta\}-\varepsilon$. In the following theorem we give the lower bound for the error of the finite element approximation of this model solution. For simplicity we shall consider only the case $\alpha+1 \geq \beta$.

Theorem 2.4. Let $\alpha>-1, \beta>-1$, and $\alpha+1 \geq \beta$. Let $u_{h} \in S_{h}(\kappa=0, \sigma)$ be the finite element approximation (with quasi-uniform meshes) of the model solution $u_{\text {model given by (2.10) }}$ with $\lambda>1-\beta-\varepsilon$. Then there holds

$$
\left\|u_{\text {model }}-u_{h}\right\|_{H_{\alpha, \beta}^{1}(\Omega)} \geq C h^{\beta+\lambda} \text {. }
$$

By Theorem 2.4 we conclude that for any $\varepsilon \in[0,1)$ there exists a function $u_{\text {model }} \in \stackrel{\circ}{H}_{\alpha, \beta}^{1}(\Omega) \cap H_{\alpha, \beta, \beta+\varepsilon}^{2}(\Omega)$ such that for any $\tilde{\varepsilon}>0$ there holds

$$
\left\|u_{\text {model }}-u_{h}\right\|_{H_{\alpha, \beta}^{1}(\Omega)} \geq C h^{(1-\varepsilon)+\tilde{\varepsilon}} \text {. }
$$

Here, $u_{h} \in S_{h}(\kappa, \sigma)$ with $\kappa=0$ is the finite element approximation of $u_{\text {model }}$. The constant $C$ in the above estimate may depend on $\tilde{\varepsilon}$. This proves that for quasiuniform meshes the error estimate (2.7) is quasi-optimal.

Now we state an inverse estimate which proves the optimality of (2.8) (in the case $\kappa=\varepsilon, \theta \leq 1 / 2$ ) and for special mesh sequences, see [2, Section 6].

Theorem 2.5. Let $\alpha>-1, \beta \geq 0, \alpha+1 \geq \beta$, and $\varepsilon \in[0,1)$. Then there exists a constant $C>0$ such that for any $u \in S_{h}(\kappa=\varepsilon, \sigma)$ and $\theta \in(0,1 / 2]$ there holds

$$
\|u\|_{X(1, \alpha, \beta, 2, \beta+\varepsilon, \theta, \infty)} \leq C h^{-\theta}\|u\|_{H_{\alpha, \beta}^{1}(\Omega)} .
$$

2.2. Applications to some other problems with degenerate and singular coefficients. In this section we study problems where the differential operator is not given in divergence form. Let us consider

$$
\begin{aligned}
-a r^{2 \beta} \Delta u+b r^{2 \alpha} u & =f \text { in } \Omega, \\
u & =0 \text { on } \partial \Omega,
\end{aligned}
$$

where $f \in L_{2, \mu}(\Omega), \alpha, \beta$, and $\mu$ are real, and $a, b$ are measurable functions satisfying the inequalities

$$
0<\underline{a} \leq a(x) \leq \bar{a}, \quad 0<\underline{b} \leq b(x) \leq \bar{b} \quad \text { almost everywhere in } \bar{\Omega} .
$$

Problems of this kind appear, e.g., in quantum mechanics when describing the motion of particles in a centrally symmetric potential field $U(r) \approx r^{s}$. In particular, our results below are applicable to the equations describing the motion of particles in the Coulomb field $\left(\beta=0, \alpha=-\frac{1}{2}\right)$ and in the field with potential energy 
$U(r) \approx r^{-2}(\beta=0, \alpha=-1)$. In the classical physical literature such problems are usually studied by an asymptotic analysis; see, e.g., [14, Chapter V and §49].

In the following we consider two cases for which one can apply the results of $\$ 2.1$ without additional restrictions on the coefficients of the equation in (2.13).

1) Let $\alpha>\beta-1$. In this case we denote

$$
b_{1}(x)=\frac{b(x)}{a(x)}, \quad f_{1}(x)=\frac{f(x)}{a(x)} r^{-2 \beta}(x) .
$$

Introducing the bilinear form

$$
A_{1}(u, v)=\int_{\Omega}\left(\nabla u \nabla v+b_{1} r^{2(\alpha-\beta)} u v\right) d x
$$

and the linear functional

$$
F_{1}(v)=\int_{\Omega} f_{1} v d x
$$

we give a weak formulation to problem (2.13):

$$
\text { find } u \in \stackrel{\circ}{H}_{\alpha-\beta, 0}^{1}(\Omega) \text { satisfying } A_{1}(u, v)=F_{1}(v) \text { for all } v \in \stackrel{\circ}{H}_{\alpha-\beta, 0}^{1}(\Omega) \text {. }
$$

Theorem 2.6. Let $\alpha>\beta-1$ and $\mu<1-2 \beta$. Then for any $f \in L_{2, \mu}(\Omega)$ there exists a unique weak solution to problem (2.13).

The finite element scheme for problem (2.13) is the same as in $\$ 2.1$ except that $A$ and $F$ in (2.5) are replaced by $A_{1}$ and $F_{1}$, respectively. One has the following analogues of Theorems 2.2 and 2.3 .

Theorem 2.7. Let $\alpha>\beta-1$, and $\varepsilon \in[0,1)$. Let $u_{h} \in S_{h}(\kappa, \sigma)$ be the finite element approximation of the solution $u$ to problem (2.13). If $u \in \stackrel{\circ}{H_{\alpha-\beta, 0}^{1}}(\Omega) \cap H_{\alpha-\beta, 0, \varepsilon}^{2}(\Omega)$, then there holds

$$
\left\|u-u_{h}\right\|_{H_{\alpha-\beta, 0}^{1}(\Omega)} \leq C \begin{cases}h\|u\|_{H_{\alpha-\beta, 0, \varepsilon}^{2}(\Omega)} & \text { if } \varepsilon \leq \kappa<1, \\ h^{\frac{1-\varepsilon}{1-\kappa}}\|u\|_{H_{\alpha-\beta, 0, \varepsilon}^{2}}(\Omega) & \text { if } 0 \leq \kappa<\varepsilon .\end{cases}
$$

Theorem 2.8. Let $\alpha>\beta-1$, and let $u \in \stackrel{\circ}{H}_{\alpha-\beta, 0}^{1}(\Omega)$ be the weak solution to problem (2.13) for the given right-hand side $f \in L_{2, \mu}(\Omega)$. Then there exists $\varepsilon \in[0,1)$ such that $u \in H_{\alpha-\beta, 0, \varepsilon}^{2}(\Omega)$ provided that $\mu \leq-2 \beta+\varepsilon$. Moreover,

$$
\|u\|_{H_{\alpha-\beta, 0, \varepsilon}^{2}(\Omega)} \leq C\|f\|_{L_{2, \mu}(\Omega)} .
$$

2) Let $\alpha=\beta-1$. For the sake of simplicity, in this case we restrict ourselves to the model problem

$$
\begin{aligned}
-\Delta u+b r^{-2} u & =f \quad \text { in } \Omega, \\
u & =0 \text { on } \partial \Omega,
\end{aligned}
$$

where $f \in L_{2, \mu}(\Omega), \mu \in \mathbb{R}$, and $b$ is a measurable function such that $0<\underline{b} \leq b(x) \leq$ $\bar{b}$ almost everywhere in $\bar{\Omega}$.

Observe that the general case of problem (2.13) with $\alpha=\beta-1$ can be reduced to (2.15) by multiplying the differential equation by $a^{-1} r^{-2 \beta}$. This situation, where $\alpha=-1$ and $\beta=0$ in (2.15), however, is not directly covered by our analysis. 
Instead, we multiply the differential equation in (2.15) by $r^{2 \varepsilon}(\varepsilon>0$ is fixed). Then, integration by parts gives the bilinear form

$$
A_{\varepsilon}(u, v)=\int_{\Omega}\left(r^{2 \varepsilon} \nabla u \nabla v+\nabla\left(r^{2 \varepsilon}\right) \nabla u v+b r^{2(\varepsilon-1)} u v\right) d x
$$

and the linear functional

$$
F_{\varepsilon}(v)=\int_{\Omega} r^{2 \varepsilon} f v d x
$$

We then formulate problem (2.15) in the following weak form:

$$
\text { find } u \in \stackrel{\circ}{H}_{\varepsilon-1, \varepsilon}^{1}(\Omega) \text { satisfying } A_{\varepsilon}(u, v)=F_{\varepsilon}(v) \text { for all } v \in \stackrel{\circ}{H}_{\varepsilon-1, \varepsilon}^{1}(\Omega) \text {. }
$$

Theorem 2.9. Let $\varepsilon \in(0, \sqrt{2 \underline{b}} / 2)$. Then, for any given $f \in L_{2,1+\varepsilon}(\Omega)$, there exists a unique weak solution $u \in \stackrel{\circ}{H}_{\varepsilon-1, \varepsilon}^{1}(\Omega)$ to problem (2.15).

Now we use the finite element scheme of $\$ 2.1$ and define the approximate solution $u_{h} \in S_{h}$ as in (2.5) with $A$ and $F$ replaced by $A_{\varepsilon}$ and $F_{\varepsilon}$, respectively. Then we have the following result on the error of the finite element approximation.

Theorem 2.10. Let $\varepsilon \in(0, \sqrt{2 \underline{b}} / 2)$ and $\tilde{\varepsilon} \in[0,1)$. Let $u_{h} \in S_{h}(\kappa, \sigma)$ be the finite element approximation of the solution $u$ to problem (2.15). If $u \in \stackrel{\circ}{H}_{\varepsilon-1, \varepsilon}^{1}$ $(\Omega) \cap H_{\varepsilon-1, \varepsilon, \varepsilon+\tilde{\varepsilon}}^{2}(\Omega)$, then

$$
\left\|u-u_{h}\right\|_{H_{\varepsilon-1, \varepsilon}^{1}(\Omega)} \leq C \begin{cases}h\|u\|_{H_{\varepsilon-1, \varepsilon, \varepsilon+\tilde{\varepsilon}}^{2}}(\Omega) & \text { if } \tilde{\varepsilon} \leq \kappa<1 \\ h^{\frac{1-\tilde{\varepsilon}}{1-\kappa}}\|u\|_{H_{\varepsilon-1, \varepsilon, \varepsilon+\tilde{\varepsilon}}^{2}(\Omega)} & \text { if } 0 \leq \kappa<\tilde{\varepsilon}\end{cases}
$$

Remark 2.1. The case $\alpha=-1, \beta=0$ considered here is not covered by Theorem 2.3. For the regularity results in this case we refer to [17].

Remark 2.2. If $\alpha<\beta-1$, then problem (2.13) could also be treated in a similar way. But in this case additional restrictions must be imposed on the elliptic operator (in particular, on the coefficient $b$ ) to prove the solvability of the corresponding variational problem.

\section{TECHNICAL RESUlts AND PROOFS OF THE MAIN THEOREMS}

Before giving the proofs of the main results we collect some auxiliary results regarding the weighted spaces $H_{\alpha, \beta}^{1}$ and $H_{\alpha, \beta, \gamma}^{2}$. We shall also use the definitions from $₫ 2$ for the standard triangle $T$ with vertices $x^{(0)}=(0,0), x^{(1)}=(1,0), x^{(2)}=$ $(1,1)$, i.e., $T=\left\{\left(x_{1}, x_{2}\right) \mid 0<x_{1}<1,0<x_{2}<x_{1}\right\}$. When it is not ambiguous we write $\|\cdot\|$ for the norm $\|\cdot\|_{L_{2}(T)}$. Denoting by $\Gamma$ a side of the triangle $T$, we shall also deal with the space $L_{2}(\Gamma)$, which is defined in an obvious way.

Lemma 3.1. Let $u$ be a function defined on the triangle $T$.

1) If $\beta<0, u$ is continuous on $\bar{T}$, and $\left\|r^{\beta}\left|D^{1} u\right|\right\|<\infty$, then there exists a constant $C>0$ depending merely on $\beta$ such that

$$
\left\|r^{\beta-1}[u-u(0,0)]\right\| \leq C\left\|r^{\beta}\left|D^{1} u\right|\right\| .
$$

2) If $\alpha>-1, \beta>0$, and $u \in H_{\alpha, \beta}^{1}(T)$, then there exists a positive constant $C$ depending on $\alpha$ and $\beta$ such that

$$
\left\|r^{\beta-1} u\right\|_{L_{2}(T)} \leq C\|u\|_{H_{\alpha, \beta}^{1}(T)} .
$$




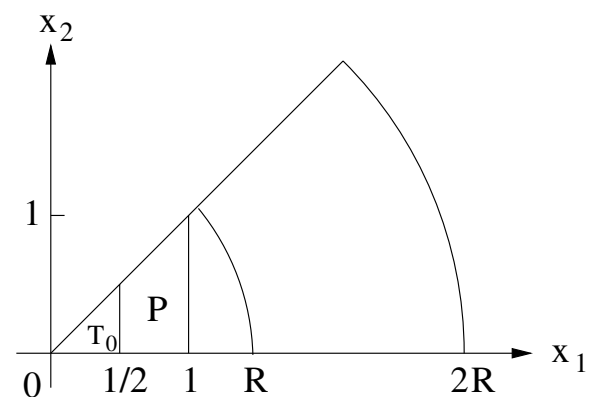

Figure 1. Notation used in the proof of Lemma 3.1 .

Proof. The first statement is proved in [2] (see Lemma 4.3).

In order to prove (3.1) we divide $T$ into the triangle

$$
T_{0}=\left\{\left(x_{1}, x_{2}\right) \mid 0 \leq x_{1} \leq 1 / 2,0 \leq x_{2} \leq x_{1}\right\}
$$

and the trapezoid $P=\left\{\left(x_{1}, x_{2}\right) \mid 1 / 2 \leq x_{1} \leq 1,0 \leq x_{2} \leq x_{1}\right\}$ (see Figure 1). Let us also introduce the finite sector $S_{R}=\{(r, \theta) \mid 0 \leq r \leq R, 0 \leq \theta \leq \pi / 4\}$, where $(r, \theta)$ are polar coordinates.

Let $R>\sqrt{2}$. Since $u \in H^{1}(P)$, there exists a smooth extension $\bar{u} \in H^{1}\left(S_{2 R} \backslash T_{0}\right)$ such that $\bar{u}(x)=u(x)$ on $P, \bar{u}(x)=0$ on $S_{2 R} \backslash S_{3 R / 2}$, and

$$
\|\bar{u}\|_{H^{1}\left(S_{2 R} \backslash T_{0}\right)} \leq C\|u\|_{H^{1}(P)},
$$

where $C>0$ is independent of $u$.

Defining $\bar{u}(x)=u(x)$ on $T_{0}$, we get a smooth extension of the function $u \in$ $H_{\alpha, \beta}^{1}(T)$ to the sector $S_{2 R}$. Using inequality (3.2) and the boundedness of $r$ and $r^{-1}$ on $S_{2 R} \backslash T_{0}$, we obtain

$$
\begin{aligned}
\|\bar{u}\|_{H_{\alpha, \beta}^{1}\left(S_{2 R}\right)}^{2} & \leq\|\bar{u}\|_{H_{\alpha, \beta}^{1}\left(T_{0}\right)}^{2}+C\|\bar{u}\|_{H^{1}\left(S_{2 R} \backslash T_{0}\right)}^{2} \\
& \leq\|u\|_{H_{\alpha, \beta}^{1}\left(T_{0}\right)}^{2}+C\|u\|_{H^{1}(P)}^{2} \leq\|u\|_{H_{\alpha, \beta}^{1}\left(T_{0}\right)}^{2}+C\|u\|_{H_{\alpha, \beta}^{1}(P)}^{2} \\
& \leq C\|u\|_{H_{\alpha, \beta}^{1}(T)}^{2} .
\end{aligned}
$$

Therefore we conclude that $\bar{u} \in H_{\alpha, \beta}^{1}\left(S_{2 R}\right)$, in particular, $\left\|r^{\beta}\left|D^{1} \bar{u}\right|\right\|_{L_{2}\left(S_{2 R}\right)}<\infty$. Since $\bar{u}(x)=0$ on the arc of the sector $S_{2 R}$, and $\beta>0$, we use the following inequality:

$$
\left\|r^{\beta-1} \bar{u}\right\|_{L_{2}\left(S_{2 R}\right)}^{2} \leq \beta^{-2}\left\|r^{\beta} \mid D^{1} \bar{u}\right\|_{L_{2}\left(S_{2 R}\right)}^{2}
$$

which stems from Hardy's inequality [12, Theorem 255] after changing variables and integrating with respect to $\theta$ (cf. [2, Lemma 4.3]).

Because $\bar{u}(x)=u(x)$ on $T=T_{0} \cup P$, we get by (3.4) and (3.3)

$$
\left\|r^{\beta-1} u\right\|_{L_{2}(T)}^{2} \leq\left\|r^{\beta-1} \bar{u}\right\|_{L_{2}\left(S_{2 R}\right)}^{2} \leq C\|\bar{u}\|_{H_{\alpha, \beta}^{1}\left(S_{2 R}\right)}^{2} \leq C\|u\|_{H_{\alpha, \beta}^{1}(T)}^{2} .
$$

In the case of a function $u$ vanishing on the boundary $\partial \Omega$ we shall need the following result. 
Lemma 3.2. Let $\beta>0$, and let $u$ be a function defined on $\Omega$ such that

$$
\left\|r^{\beta}\left|D^{1} u\right|\right\|_{L_{2}(\Omega)}<\infty .
$$

If $u$ vanishes on the boundary $\partial \Omega$, then

$$
\left\|r^{\beta-1} u\right\|_{L_{2}(\Omega)} \leq \beta^{-1}\left\|r^{\beta}\left|D^{1} u\right|\right\|_{L_{2}(\Omega)} .
$$

Proof. Let us consider the unit disk $B$ with center at $O$. We have $\bar{\Omega} \subset B$. Extending the function $u$ by zero on $B$ and applying inequality (3.4) to the extension $\bar{u}$ on $B$, we get the result.

Lemma 3.3. Let $\alpha>-1, \beta>-1$, and $-1<\gamma<1$. If $u \in H_{\alpha, \beta, \gamma}^{2}(T)$, then $u$ is continuous on $\bar{T}$, and

$$
\|u\|_{C^{0}(\bar{T})} \leq C\|u\|_{H_{\alpha, \beta, \gamma}^{2}(T)},
$$

with $C>0$ being independent of $u$.

Proof. Let $u \in H_{\alpha, \beta, \gamma}^{2}(T)$, and assume first that $\gamma \in[0,1)$. Applying statement 2) of Lemma 3.1 to the functions $u$ and $\frac{\partial u}{\partial x_{i}}(i=1,2)$, we obtain

$$
\begin{gathered}
\|u\|^{2} \leq C\left(\left\|r^{\alpha} u\right\|^{2}+\left\|r\left|D^{1} u\right|\right\|^{2}\right) \leq C\left(\left\|r^{\alpha} u\right\|^{2}+\left\|D^{1} u\right\|^{2}\right), \\
\left\|D^{1} u\right\|^{2}=\sum_{i=1}^{2}\left\|\frac{\partial u}{\partial x_{i}}\right\|^{2} \leq C\left[\sum_{i=1}^{2}\left\|r^{\beta} \frac{\partial u}{\partial x_{i}}\right\|^{2}+\left\|r\left|D^{2} u\right|\right\|^{2}\right] \\
\leq C\left[\left\|r^{\beta}\left|D^{1} u\right|\right\|^{2}+\left\|r^{\gamma}\left|D^{2} u\right|\right\|^{2}\right] .
\end{gathered}
$$

Hence

$$
\|u\|_{H_{\gamma}^{2,2}(T)}^{2}=\|u\|^{2}+\left\|D^{1} u\right\|^{2}+\left\|r^{\gamma}\left|D^{2} u\right|\right\|^{2} \leq C\|u\|_{H_{\alpha, \beta, \gamma}^{2}(T)}^{2},
$$

and therefore $u \in H_{\gamma}^{2,2}(T)$ with $\gamma \in[0,1)$.

Since $H_{\gamma}^{2,2}(T) \subset C^{0}(\bar{T})$ by (2.1), and $\|u\|_{C^{0}(\bar{T})} \leq C\|u\|_{H_{\gamma}^{2,2}(T)}$, we conclude that $u \in C^{0}(\bar{T})$, and inequality (3.5) holds.

If $\gamma \in(-1,0)$, then the statement of the lemma is also true, because in this case $H_{\alpha, \beta, \gamma}^{2}(T) \subset H_{\alpha, \beta, 0}^{2}(T) \subset C^{0}(\bar{T})$ and

$$
\|u\|_{C^{0}(\bar{T})} \leq C\|u\|_{H_{\alpha, \beta, 0}^{2}(T)} \leq C\|u\|_{H_{\alpha, \beta, \gamma}^{2}(T)} .
$$

Lemma 3.4. Let $\alpha>-1, \beta>-1$, and $-1<\gamma<1$. If $u \in H_{\alpha, \beta, \gamma}^{2}(\Omega)$, then $u$ is continuous on $\bar{\Omega}$, and

$$
\|u\|_{C^{0}(\bar{\Omega})} \leq C\|u\|_{H_{\alpha, \beta, \gamma}^{2}(\Omega)} .
$$

The proof is similar to that of Lemma 3.3

Lemma 3.5. Let $\gamma>0, \gamma \neq 1$, and let $u \in H_{\alpha, \beta, \gamma}^{2}(T)$; if $\gamma \in(0,1)$, assume additionally that $u(0,0)=0$. Then for any $\varepsilon \in[0,1)$ one has $v=r^{\gamma-\varepsilon} u \in H_{\varepsilon}^{2,2}(T)$, $v(0,0)=0$, and

$$
\|v\|_{H_{\varepsilon}^{2,2}(T)} \leq C\|u\|_{H_{\alpha, \beta, \gamma}^{2}(T)}
$$

with a constant $C>0$ which is independent of $u$. 
Proof. Differentiating the function $v=r^{\gamma-\varepsilon} u$ with respect to polar coordinates $(r, \theta) \in T$, we find

$$
\begin{gathered}
\|v\|^{2}=\left\|r^{\gamma-\varepsilon} u\right\|^{2} \\
\left\|D^{1} v\right\|^{2} \leq C\left(\left\|r^{\gamma-\varepsilon-1} u\right\|^{2}+\left\|r^{\gamma-\varepsilon}\left|D^{1} u\right|\right\|^{2}\right), \\
\left\|r^{\varepsilon}\left|D^{2} v\right|\right\|^{2} \leq C\left(\left\|r^{\gamma-2} u\right\|^{2}+\left\|r^{\gamma-1}\left|D^{1} u\right|\right\|^{2}+\left\|r^{\gamma}\left|D^{2} u\right|\right\|^{2}\right) .
\end{gathered}
$$

Then for any $\varepsilon \in[0,1)$

$$
\|v\|_{H_{\varepsilon}^{2,2}(T)}^{2} \leq C\left(\left\|r^{\gamma-2} u\right\|^{2}+\left\|r^{\gamma-1}\left|D^{1} u\right|\right\|^{2}+\left\|r^{\gamma}\left|D^{2} u\right|\right\|^{2}\right) .
$$

Let $\gamma \in(0,1)$. Since in this case $u(0,0)=0$ by assumption, and $u$ is continuous on $\bar{T}$ by Lemma 3.3. we successively apply statements 1 ) and 2) of Lemma 3.1 to obtain

$$
\left\|r^{\gamma-2} u\right\|^{2} \leq C\left\|r^{\gamma-1}\left|D^{1} u\right|\right\|^{2} \leq C\left(\left\|r^{\alpha} u\right\|^{2}+\left\|r^{\gamma-1}\left|D^{1} u\right|\right\|^{2}\right)
$$

and

$$
\left\|r^{\gamma-1}\left|D^{1} u\right|\right\|^{2} \leq C\left(\left\|r^{\beta}\left|D^{1} u\right|\right\|^{2}+\left\|r^{\gamma}\left|D^{2} u\right|\right\|^{2}\right) .
$$

The analogous inequalities are also valid for $\gamma>1$. To prove them in that case it is enough to use statement 2) of Lemma 3.1 twice.

Now, by inequality (3.7), we conclude that $v \in H_{\varepsilon}^{2,2}(T)$, and (3.6) holds. In order to prove that $v(0,0)=0$ for any $\varepsilon \in[0,1)$ we take $\varepsilon_{1}, \varepsilon_{2} \in(0,1)$ such that $\varepsilon=\varepsilon_{1}-\varepsilon_{2}$. It has been established above that $r^{\gamma-\varepsilon_{1}} u \in H_{\varepsilon_{1}}^{2,2}(T) \subset C^{0}(\bar{T})$ (see Lemma 3.3). Therefore $v(0,0)=\left(r^{\gamma-\varepsilon} u\right)(0,0)=\left(r^{\gamma-\varepsilon_{1}} u\right)(0,0) r^{\varepsilon_{2}}(0,0)=0$.

Remark 3.1. If $\gamma=0$ or $\gamma=1$, then the statement of Lemma 3.5 in general is not true. In both these cases it is necessary to introduce a small parameter $\varepsilon_{0}>0$ such that $\varepsilon+\varepsilon_{0}<1$ for $\varepsilon \in[0,1)$. Then we consider $\|v\|_{H_{\varepsilon+\varepsilon_{0}}^{2,2}(T)}$ instead of $\|v\|_{H_{\varepsilon}^{2,2}(T)}$ with $v$ from before. Because $u \in H_{\alpha, \beta, \gamma}^{2}(T) \subset H_{\alpha, \beta, \gamma+\varepsilon_{0}}^{2}(T)$, we apply Lemma 3.5 (assuming $u(0,0)=0$ for $\gamma=0$ ) and establish that $v=r^{\gamma-\varepsilon} u=r^{\left(\gamma+\varepsilon_{0}\right)-\left(\varepsilon+\varepsilon_{0}\right)} u \in$ $H_{\varepsilon+\varepsilon_{0}}^{2,2}(T), v(0,0)=0$, and $\|v\|_{H_{\varepsilon+\varepsilon_{0}}^{2,2}(T)} \leq C\|u\|_{H_{\alpha, \beta, \gamma}^{2}(T)}$ for any $\varepsilon \in[0,1)$ and sufficiently small $\varepsilon_{0}>0$.

Lemma 3.6. Let $\alpha>-1, \beta>-1$, and $0 \leq \gamma<\min \{\alpha+1, \beta\}+1$. Then the space $H_{\alpha, \beta, \gamma}^{2}(T)$ is compactly imbedded in the space $H_{\alpha, \beta}^{1}(T)$.

Proof. Let $\left\{u_{j}\right\}_{j=1}^{\infty}$ be a bounded sequence in $H_{\alpha, \beta, \gamma}^{2}(T)$, and suppose that $u_{j}(0,0)=0$ for $\gamma \in[0,1)$. If $\gamma>0$, and $\gamma \neq 1$, then, taking $v_{j}=r^{\gamma-\varepsilon} u_{j}$ and using Lemma 3.5, we get a bounded sequence $\left\{v_{j}\right\}_{j=1}^{\infty}$ in $H_{\varepsilon}^{2,2}(T)$ for any $\varepsilon \in[0,1)$. By Lemma 3.4 of [11, the space $H_{\varepsilon}^{2,2}(T)$ is compactly imbedded in $H^{1}(T)$. Therefore there exists a subsequence, denoted again by $\left\{v_{j}\right\}_{j=1}^{\infty}$, which converges to a function $\tilde{v} \in H^{1}(T)$. This conclusion is also true when $\gamma=0$ and $\gamma=1$. In fact, $\left\{v_{j}\right\}_{j=1}^{\infty}$ is bounded in $H_{\varepsilon+\varepsilon_{0}}^{2,2}(T)$ for $0<\varepsilon+\varepsilon_{0}<1$ (see Remark 3.1), and $H_{\varepsilon+\varepsilon_{0}}^{2,2}(T) \stackrel{c}{\subset} H^{1}(T)$.

Now for $0 \leq \gamma<\min \{\alpha+1, \beta\}+1$ we denote $\tilde{u}=r^{-\gamma+\varepsilon} \tilde{v}$. Then for $i=1,2$ one has

$$
\left|\frac{\partial \tilde{u}(x)}{\partial x_{i}}\right| \leq \max \{1,|\gamma-\varepsilon|\}\left(r^{-\gamma-1+\varepsilon}|\tilde{v}(x)|+r^{-\gamma+\varepsilon}\left|\frac{\partial \tilde{v}(x)}{\partial x_{i}}\right|\right) .
$$


Hence

$$
\begin{gathered}
\left\|r^{\alpha} \tilde{u}\right\|^{2}=\left\|r^{\alpha-\gamma+\varepsilon} \tilde{v}\right\|^{2}, \\
\left\|r^{\beta}\left|D^{1} \tilde{u}\right|\right\|^{2} \leq C\left(\left\|r^{\beta-\gamma-1+\varepsilon} \tilde{v}\right\|^{2}+\left\|r^{\beta-\gamma+\varepsilon}\left|D^{1} \tilde{v}\right|\right\|^{2}\right) .
\end{gathered}
$$

Since $\alpha>-1, \beta>-1$, and $0 \leq \gamma<\min \{\alpha+1, \beta\}+1$, there exists $\varepsilon \in[0,1)$ such that $0 \leq \gamma<\min \{\alpha+1, \beta\}+\varepsilon$, and thereby, $\alpha+1-\gamma+\varepsilon>0$ and $\beta-\gamma+\varepsilon>0$. Taking such an $\varepsilon$ in the above arguments and using statement 2) of Lemma 3.1 we deduce from (3.8) that

$$
\begin{gathered}
\left\|r^{\alpha} \tilde{u}\right\|^{2} \leq C\left(\|\tilde{v}\|^{2}+\left\|r^{\alpha+1-\gamma+\varepsilon}\left|D^{1} \tilde{v}\right|\right\|^{2}\right) \leq C\|\tilde{v}\|_{H^{1}(T)}^{2}, \\
\left\|r^{\beta}\left|D^{1} \tilde{u}\right|\right\|^{2} \leq C\left(\|\tilde{v}\|^{2}+\left\|r^{\beta-\gamma+\varepsilon}\left|D^{1} \tilde{v}\right|\right\|^{2}\right) \leq C\|\tilde{v}\|_{H^{1}(T)}^{2} .
\end{gathered}
$$

Therefore $\tilde{u} \in H_{\alpha, \beta}^{1}(T)$. Similarly we have

$$
\left\|u_{j}-\tilde{u}\right\|_{H_{\alpha, \beta}^{1}(T)}=\left\|r^{-\gamma+\varepsilon}\left(v_{j}-\tilde{v}\right)\right\|_{H_{\alpha, \beta}^{1}(T)} \leq C\left\|v_{j}-\tilde{v}\right\|_{H^{1}(T)} \rightarrow 0 \text { as } j \rightarrow \infty .
$$

It remains to consider the case when $\gamma \in[0,1)$ and $u_{j}(0,0)=$ const $\neq 0$. Let us define $\bar{u}_{j}(x)=u_{j}(x)-u_{j}(0,0)$. Using Lemma 3.3 we obtain

$$
\begin{gathered}
\left|u_{j}(0,0)\right| \leq C\left\|u_{j}\right\|_{H_{\alpha, \beta, \gamma}^{2}(T)}, \\
\left\|\bar{u}_{j}\right\|_{H_{\alpha, \beta, \gamma}^{2}(T)} \leq\left\|u_{j}\right\|_{H_{\alpha, \beta, \gamma}^{2}(T)}+C(\alpha)\left|u_{j}(0,0)\right| \leq C\left\|u_{j}\right\|_{H_{\alpha, \beta, \gamma}^{2}(T)} .
\end{gathered}
$$

Thus $\bar{u}_{j} \in H_{\alpha, \beta, \gamma}^{2}(T)$ and $\bar{u}_{j}(0,0)=0$. Using the boundedness of $\left\{u_{j}(0,0)\right\}_{j=1}^{\infty}$ and the proof for the case $u_{j}(0,0)=0$, we find a subsequence $\left\{u_{j}\right\}_{j=1}^{\infty}$, a real number $A$, and a function $\tilde{u} \in H_{\alpha, \beta}^{1}(T)$ such that $u_{j}(0,0) \rightarrow A$, and $\bar{u}_{j}=u_{j}-u_{j}(0,0) \rightarrow \tilde{u}$ in $H_{\alpha, \beta}^{1}(T)$ as $j \rightarrow \infty$. Then

$$
\left\|u_{j}-(\tilde{u}+A)\right\|_{H_{\alpha, \beta}^{1}(T)} \leq\left\|\bar{u}_{j}-\tilde{u}\right\|_{H_{\alpha, \beta}^{1}(T)}+\left\|u_{j}(0,0)-A\right\|_{H_{\alpha, \beta}^{1}(T)} \rightarrow 0 \quad \text { as } \quad j \rightarrow \infty .
$$

This completes the proof.

Lemma 3.7. Let $T$ be the standard triangle with vertices $x^{(l)}(l=0,1,2)$, and let $x^{(3)}$ be an interior point of T. Assume that $\alpha>-1, \beta>-1$, and $0 \leq \gamma<$ $\min \{\alpha+1, \beta\}+1$. If $u \in H_{\alpha, \beta, \gamma}^{2}(T)$, then there exists a constant $C>0$ independent of $u$ such that

$$
\|u\|_{H_{\alpha, \beta, \gamma}^{2}(T)}^{2} \leq C\left(\left\|r^{\gamma}\left|D^{2} u\right|\right\|_{L_{2}(T)}^{2}+\sum_{l=1}^{3}\left|u\left(x^{(l)}\right)\right|^{2}\right) .
$$

Proof. This statement is proved by making use of standard arguments relying on the compactness property in Lemma 3.6 (see, e.g., [6]).

In the following let $\Gamma$ be the side $\left\{\left(x_{1}, x_{2}\right) \mid 0<x_{1}<1, x_{2}=0\right\}$ of the standard triangle $T$.

Lemma 3.8. Let $\alpha>-1, \beta>0$, and $u \in H_{\alpha, \beta}^{1}(T)$. Then

$$
\left\|r^{\beta-1 / 2} u\right\|_{L_{2}(\Gamma)} \leq C\|u\|_{H_{\alpha, \beta}^{1}(T)} .
$$


Proof. Considering a sufficiently smooth function $u$ defined on $T$, we have

$$
u\left(\bar{x}_{1}, 0\right)=u\left(\bar{x}_{1}, x_{2}\right)-\int_{0}^{x_{2}} \frac{\partial u\left(\bar{x}_{1}, \widetilde{x}_{2}\right)}{\partial \widetilde{x}_{2}} d \widetilde{x}_{2}, \quad \bar{x}_{1} \in(0,1), \quad x_{2} \in\left(0, \bar{x}_{1}\right) .
$$

Hence, by the Schwarz inequality,

$$
u^{2}\left(\bar{x}_{1}, 0\right) \leq C\left(u^{2}\left(\bar{x}_{1}, x_{2}\right)+x_{2} \int_{0}^{\bar{x}_{1}}\left|\frac{\partial u\left(\bar{x}_{1}, x_{2}\right)}{\partial x_{2}}\right|^{2} d x_{2}\right) .
$$

Multiplying (3.11) by $\bar{x}_{1}^{2(\beta-1)}$ for $\beta>0$ and integrating first with respect to $x_{2} \in$ $\left(0, \bar{x}_{1}\right)$, and then with respect to $\bar{x}_{1} \in(0,1)$, we obtain

$$
\begin{aligned}
\left\|r^{\beta-1 / 2} u\right\|_{L_{2}(\Gamma)}^{2} & \leq C\left(\left\|x_{1}^{\beta-1} u\right\|_{L_{2}(T)}^{2}+\left\|x_{1}^{\beta}\left|D^{1} u\right|\right\|_{L_{2}(T)}^{2}\right) \\
& \leq C\left(\left\|r^{\beta-1} u\right\|_{L_{2}(T)}^{2}+\left\|r^{\beta}\left|D^{1} u\right|\right\|_{L_{2}(T)}^{2}\right),
\end{aligned}
$$

because $x_{1} \leq r=\sqrt{x_{1}^{2}+x_{2}^{2}} \leq x_{1} \sqrt{2}$ on $T$.

It remains to apply inequality (3.1) and standard density arguments to get (3.10).

Proof of Theorem 2.1. Using assumptions (1.2) and the Schwarz inequality, we obtain the continuity and $\stackrel{\circ}{H}_{\alpha, \beta}^{1}(\Omega)$-ellipticity of the bilinear form $A(u, v)$.

If $\beta>-1$ and $\mu=-\alpha$, then we get the continuity of the linear functional by applying the Schwarz inequality:

$$
|F(v)| \leq \int_{\Omega}\left|r^{\mu} f\right|\left|r^{\alpha} v\right| d x \leq\|f\|_{L_{2, \mu}(\Omega)}\|v\|_{H_{\alpha, \beta}^{1}(\Omega)}, \quad v \in \stackrel{\circ}{H}_{\alpha, \beta}^{1}(\Omega) .
$$

If $\beta>0$ and $\mu=1-\beta$, we additionally use Lemma 3.2 and obtain

$$
\begin{aligned}
|F(v)| & \leq \int_{\Omega}\left|r^{\mu} f\right|\left|r^{\beta-1} v\right| d x \leq\|f\|_{L_{2, \mu}(\Omega)}\left\|r^{\beta-1} v\right\|_{L_{2}(\Omega)} \\
& \leq C\|f\|_{L_{2, \mu}(\Omega)}\left\|r^{\beta}\left|D^{1} v\right|\right\|_{L_{2}(\Omega)} \leq C\|f\|_{L_{2, \mu}(\Omega)}\|v\|_{H_{\alpha, \beta}^{1}(\Omega)}, \quad v \in \stackrel{\circ}{H}_{\alpha, \beta}^{1}(\Omega) .
\end{aligned}
$$

If $\beta=0$ and $\mu<1$, then there exists $\varepsilon>0$ such that $\mu \leq 1-\varepsilon$, and similarly as above we estimate

$$
\begin{aligned}
|F(v)| \leq C\|f\|_{L_{2, \mu}(\Omega)}\left\|r^{\varepsilon-1} v\right\|_{L_{2}(\Omega)} & \leq C\|f\|_{L_{2, \mu}(\Omega)}\left\|r^{\varepsilon}\left|D^{1} v\right|\right\|_{L_{2}(\Omega)} \\
& \leq C\|f\|_{L_{2, \mu}(\Omega)}\|v\|_{H_{\alpha, 0}^{1}(\Omega)}, \quad v \in \stackrel{\circ}{H}_{\alpha, 0}^{1}(\Omega) .
\end{aligned}
$$

Now applying the Lax-Milgram lemma we establish the existence and uniqueness of a solution $u \in \stackrel{\circ}{H}_{\alpha, \beta}^{1}(\Omega)$ to (1.1), and inequality (2.3) follows.

In order to prove Theorem 2.2 we shall obtain estimate (2.7) separately for the finite elements having the origin as a vertex, and for the elements separated from the singularity point $O$.

Let $\alpha>-1, \beta>-1$, and assume that the weak solution $u$ to problem (1.1) belongs to the space $\stackrel{\circ}{H}_{\alpha, \beta}^{1}(\Omega) \cap H_{\alpha, \beta, \gamma}^{2}(\Omega)$ with $\gamma=\min \{\alpha+1, \beta\}+\varepsilon$ for some $\varepsilon \in[0,1)$. We shall denote by $\Omega_{0}$ the patch of the elements which have the origin $O$ as a vertex. Obviously $\Omega_{0}$ is a polygon, and $O$ is an interior point. 


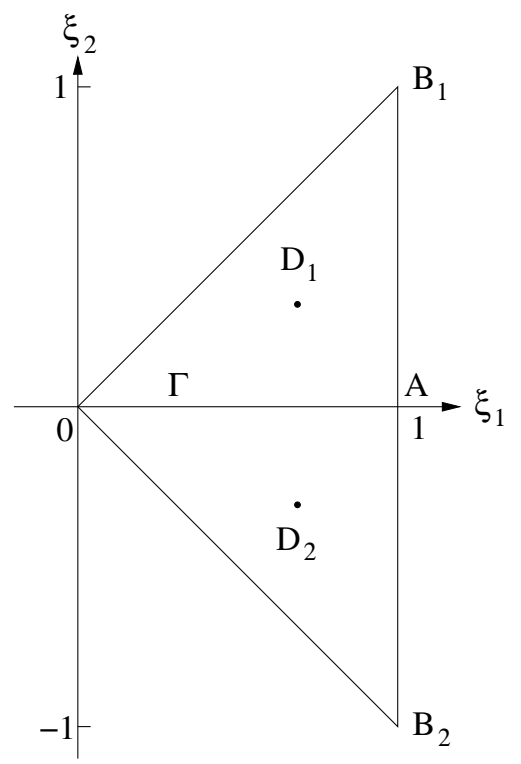

Figure 2. Notation used in the proof of Lemma 3.9.

Lemma 3.9. If $u \in H_{\alpha, \beta, \gamma}^{2}\left(\Omega_{0}\right)$, then there exists a continuous function $v$ defined on $\Omega_{0}$ such that $v=u$ at the vertices of $\Omega_{0}, v$ is linear on each finite element $\Omega_{i} \subset \Omega_{0}$, and

$$
\|u-v\|_{H_{\alpha, \beta}^{1}\left(\Omega_{0}\right)}^{2} \leq C \sum_{i: \Omega_{i} \subset \Omega_{0}} h_{i}^{2(1-\varepsilon)}\left\|r^{\gamma} \mid D^{2} u\right\|_{L_{2}\left(\Omega_{i}\right)}^{2} .
$$

Proof. Assume first that $\gamma \geq 0$. Let us consider two standard triangles $T_{1}=O A B_{1}$ and $T_{2}=O A B_{2}$, and let $D_{i}(i=1,2)$ be an interior point of $T_{i}$ (see Figure 2). If $U \in H_{\alpha, \beta, \gamma}^{2}\left(T_{1} \cup T_{2}\right)$, then $U$ is continuous on $\overline{T_{1} \cup T_{2}} \backslash\{O\}$, and for $i=1,2$ there exists a linear function $\widetilde{V}_{i}$ interpolating $U$ at the points $A, B_{i}, D_{i}$. Applying Lemma 3.7 to the function $\left(U-\widetilde{V}_{i}\right)$, we obtain for $i=1,2$

$$
\left\|U-\widetilde{V}_{i}\right\|_{H_{\alpha, \beta, \gamma}^{2}\left(T_{i}\right)}^{2} \leq C\left\|r^{\gamma}\left|D^{2}\left(U-\widetilde{V}_{i}\right)\right|\right\|_{L_{2}\left(T_{i}\right)}^{2}=C\left\|r^{\gamma}\left|D^{2} U\right|\right\|_{L_{2}\left(T_{i}\right)}^{2}
$$

and

$$
\left\|U-\widetilde{V}_{i}\right\|_{H_{\alpha, \beta}^{1}\left(T_{i}\right)}^{2} \leq\left\|U-\widetilde{V}_{i}\right\|_{H_{\alpha, \beta, \gamma}^{2}\left(T_{i}\right)}^{2} \leq C\left\|r^{\gamma}\left|D^{2} U\right|\right\|_{L_{2}\left(T_{i}\right)}^{2} .
$$

Let $\Gamma=\left\{\left(\xi_{1}, \xi_{2}\right) \mid 0<\xi_{1}<1, \xi_{2}=0\right\}$ be the common side of $T_{1}$ and $T_{2}$. We shall modify the functions $\widetilde{V}_{i}(i=1,2)$ in order to obtain a continuous piecewise linear function $V$ approximating $U$ on $T_{1} \cup T_{2}$.

Denote

$$
w\left(\xi_{1}\right)=\left.\left[\widetilde{V}_{1}(\xi)-\widetilde{V}_{2}(\xi)\right]\right|_{\Gamma}, \quad \xi_{1} \in[0,1] .
$$

Observing that $w(1)=0$, we find a linear polynomial $W(\xi)$ defined on $T_{2}$ such that

$$
W(1,-1)=0,\left.\quad W(\xi)\right|_{\Gamma}=w\left(\xi_{1}\right),
$$


and

$$
\|W\|_{H_{\alpha, \beta}^{1}\left(T_{2}\right)}^{2} \leq C\left\|r^{\beta+1 / 2}\left|D^{1} w\right|\right\|_{L_{2}(\Gamma)}^{2} \leq C \sum_{i=1}^{2}\left\|r^{\beta+1 / 2}\left|D^{1}\left(U-\widetilde{V}_{i}\right)\right|\right\|_{L_{2}(\Gamma)}^{2},
$$

with $C>0$ depending only on $\alpha$ and $\beta$.

Applying Lemma 3.8 to each function $\frac{\partial}{\partial x_{j}}\left(U-\widetilde{V}_{i}\right)(j=1,2)$ we get for $i=1,2$

$$
\begin{aligned}
& \left\|r^{\beta+1 / 2}\left|D^{1}\left(U-\widetilde{V}_{i}\right)\right|\right\|_{L_{2}(\Gamma)}^{2} \\
& \quad \leq C\left(\left\|r^{\beta}\left|D^{1}\left(U-\widetilde{V}_{i}\right)\right|\right\|_{L_{2}\left(T_{i}\right)}^{2}+\left\|r^{\beta+1}\left|D^{2}\left(U-\widetilde{V}_{i}\right)\right|\right\|_{L_{2}\left(T_{i}\right)}^{2}\right),
\end{aligned}
$$

and, because $\gamma=\min \{\alpha+1, \beta\}+\varepsilon<\beta+1$,

$$
\leq C\left(\left\|r^{\beta}\left|D^{1}\left(U-\widetilde{V}_{i}\right)\right|\right\|_{L_{2}\left(T_{i}\right)}^{2}+\left\|r^{\gamma}\left|D^{2}\left(U-\widetilde{V}_{i}\right)\right|\right\|_{L_{2}\left(T_{i}\right)}^{2}\right) .
$$

Hence by (3.15) we have

$$
\|W\|_{H_{\alpha, \beta}^{1}\left(T_{2}\right)}^{2} \leq C \sum_{i=1}^{2}\left\|U-\widetilde{V}_{i}\right\|_{H_{\alpha, \beta, \gamma}^{2}\left(T_{i}\right)}^{2} .
$$

Now we set $V_{1}(\xi)=\widetilde{V}_{1}(\xi)$ for $\xi \in T_{1}$, and $V_{2}(\xi)=\widetilde{V}_{2}(\xi)+W(\xi)$ for $\xi \in T_{2}$. Then we deduce from (3.13), (3.14), and (3.16)

$$
\begin{aligned}
\left\|U-V_{1}\right\|_{H_{\alpha, \beta}^{1}\left(T_{1}\right)}^{2} & =\left\|U-\widetilde{V}_{1}\right\|_{H_{\alpha, \beta}^{1}\left(T_{1}\right)}^{2} \leq C\left\|r^{\gamma}\left|D^{2} U\right|\right\|_{L_{2}\left(T_{1}\right)}^{2} \\
\left\|U-V_{2}\right\|_{H_{\alpha, \beta}^{1}\left(T_{2}\right)}^{2} & \leq C\left(\left\|U-\widetilde{V}_{2}\right\|_{H_{\alpha, \beta}^{1}\left(T_{2}\right)}^{2}+\|W\|_{H_{\alpha, \beta}^{1}\left(T_{2}\right)}^{2}\right) \\
& \leq C \sum_{i=1}^{2}\left\|r^{\gamma}\left|D^{2} U\right|\right\|_{L_{2}\left(T_{i}\right)}^{2}
\end{aligned}
$$

moreover $\left.V_{1}(\xi)\right|_{\Gamma}=\left.V_{2}(\xi)\right|_{\Gamma}$.

Using the above arguments and linear transformations $M_{i}: T_{1} \rightarrow \bar{\Omega}_{i}$ (or, $\widetilde{M}_{i}$ : $\left.T_{2} \rightarrow \bar{\Omega}_{i}\right)$ for each finite element $\Omega_{i} \subset \Omega_{0}$, we construct a continuous function $v$ defined on $\bar{\Omega}_{0}$ such that $v=u$ at the vertices of $\Omega_{0}$, and the restrictions $v_{i}=\left.v\right|_{\Omega_{i}}$ are linear polynomials. Furthermore, applying standard scaling arguments and recalling that $\gamma=\min \{\alpha+1, \beta\}+\varepsilon$, we get from (3.17)

$$
\|u-v\|_{H_{\alpha, \beta}^{1}\left(\Omega_{0}\right)}^{2}=\sum_{i: \Omega_{i} \subset \Omega_{0}}\left\|u-v_{i}\right\|_{H_{\alpha, \beta}^{1}\left(\Omega_{i}\right)}^{2} \leq C \sum_{i: \Omega_{i} \subset \Omega_{0}} h_{i}^{2(1-\varepsilon)}\left\|r^{\gamma}\left|D^{2} u\right|\right\|_{L_{2}\left(\Omega_{i}\right)}^{2} .
$$

Then estimate (3.12) follows for $\gamma \geq 0$. In particular, for $\beta \in(-1,0)$ and $\varepsilon=-\beta \in$ $(0,1)$ we have $\gamma=\min \{\alpha+1, \beta\}+\varepsilon=0$, and the previous estimate yields

$$
\|u-v\|_{H_{\alpha, \beta}^{1}\left(\Omega_{0}\right)}^{2} \leq C \sum_{i: \Omega_{i} \subset \Omega_{0}} h_{i}^{2(1+\beta)}\left\|D^{2} u\right\|_{L_{2}\left(\Omega_{i}\right)}^{2}
$$

provided that $u \in H_{\alpha, \beta, 0}^{2}\left(\Omega_{0}\right)$.

Observe that $\gamma \in(-1,0)$ if and only if $\beta \in(-1,0)$ and $\varepsilon \in[0,-\beta)$. Moreover, $\gamma=\beta+\varepsilon$. In this case, if $u \in H_{\alpha, \beta, \gamma}^{2}\left(\Omega_{0}\right)$, then $u \in H_{\alpha, \beta, 0}^{2}\left(\Omega_{0}\right)$, and we obtain by 
the previous estimate

$$
\begin{aligned}
\|u-v\|_{H_{\alpha, \beta}^{1}\left(\Omega_{0}\right)}^{2} & \leq C \sum_{i: \Omega_{i} \subset \Omega_{0}} h_{i}^{2(1+\beta)}\left\|D^{2} u\right\|_{L_{2}\left(\Omega_{i}\right)}^{2} \\
& \leq C \sum_{i: \Omega_{i} \subset \Omega_{0}} h_{i}^{2(1+\beta)} h_{i}^{-2(\beta+\varepsilon)}\left\|r^{\gamma}\left|D^{2} u\right|\right\|_{L_{2}\left(\Omega_{i}\right)}^{2} \\
& =C \sum_{i: \Omega_{i} \subset \Omega_{0}} h_{i}^{2(1-\varepsilon)}\left\|r^{\gamma}\left|D^{2} u\right|\right\|_{L_{2}\left(\Omega_{i}\right)}^{2} .
\end{aligned}
$$

This finishes the proof.

Proof of Theorem 2.2. By Cea's lemma (see (2.6)) we only need to construct a piecewise polynomial $v \in S_{h}(\kappa, \sigma)$ that satisfies (2.7). We divide this construction into three parts: the approximation close to the singularity and the approximation away from the singularity for quasi-uniform meshes (i) and graded meshes (ii).

Approximation on $\Omega_{0}$ (close to the singularity). By Lemma 3.9 we find a piecewise polynomial $v_{0}$ that interpolates $u$ at all vertices of $\Omega_{0}$ except $O$ such that there holds

$$
\left\|u-v_{0}\right\|_{H_{\alpha, \beta}^{1}\left(\Omega_{0}\right)}^{2} \leq C \sum_{j: \Omega_{j} \subset \Omega_{0}} h_{j}^{2(1-\varepsilon)}\left\|r^{\gamma}\left|D^{2} u\right|\right\|_{L_{2}\left(\Omega_{j}\right)}^{2} .
$$

Using the definition of the graded mesh for elements $\Omega_{j} \subset \Omega_{0}$ one has

$$
\begin{gathered}
h_{j} \leq \sigma^{-1} h \sup _{x \in \Omega_{j} \subset \Omega_{0}} r^{\kappa}(x) \leq C h h_{j}^{\kappa}, \\
h_{j} \geq \sigma h \sup _{x \in \Omega_{j} \subset \Omega_{0}} r^{\kappa}(x) \geq C h h_{j}^{\kappa} .
\end{gathered}
$$

Estimates (3.18) and (3.19) imply for any $\kappa \in[0,1)$

$$
\left\|u-v_{0}\right\|_{H_{\alpha, \beta}^{1}\left(\Omega_{0}\right)}^{2} \leq C h^{\frac{2(1-\varepsilon)}{1-\kappa}}\left\|r^{\gamma}\left|D^{2} u\right|\right\|_{L_{2}\left(\Omega_{0}\right)}^{2}
$$

Approximation on elements separated from the singularity. For elements $\Omega_{i}$ separated from the singularity point we use standard interpolation results to find a linear polynomial $v_{i}$ that interpolates $u$ at the vertices of $\Omega_{i}$ such that

$$
\begin{gathered}
\left\|u-v_{i}\right\|_{L_{2}\left(\Omega_{i}\right)}^{2} \leq C h_{i}^{4}\left\|D^{2} u\right\|_{L_{2}\left(\Omega_{i}\right)}^{2}, \\
\left\|D^{1}\left(u-v_{i}\right)\right\|_{L_{2}\left(\Omega_{i}\right)}^{2} \leq C h_{i}^{2}\left\|D^{2} u\right\|_{L_{2}\left(\Omega_{i}\right)}^{2} .
\end{gathered}
$$

For elements $\Omega_{i}$ with $O \notin \bar{\Omega}_{i}$ one finds that there holds

$$
C_{1} h^{\frac{1}{1-\kappa}} \leq h_{i} \leq C_{2} h .
$$

(i) Case $\kappa=0$ (quasi-uniform meshes). Recalling that $\alpha+1>0, \gamma \leq \alpha+1+\varepsilon$, and $d_{i} \leq r \leq d_{i}+C h_{i}$ on $\Omega_{i}$, we deduce from (3.22) that

$$
\begin{aligned}
\left\|r^{\alpha}\left(u-v_{i}\right)\right\|_{L_{2}\left(\Omega_{i}\right)}^{2} & \leq \frac{\left(d_{i}+C h_{i}\right)^{2 \alpha+2}}{d_{i}^{2}}\left\|u-v_{i}\right\|_{L_{2}\left(\Omega_{i}\right)}^{2} \\
& \leq C \frac{\left(d_{i}+C h_{i}\right)^{2 \alpha+2}}{d_{i}^{2}} h_{i}^{4}\left\|D^{2} u\right\|_{L_{2}\left(\Omega_{i}\right)}^{2} \\
& \leq C\left(\frac{d_{i}+C h_{i}}{d_{i}}\right)^{2(\alpha+2+\varepsilon)} \frac{h_{i}^{4}}{\left(d_{i}+C h_{i}\right)^{2(1+\varepsilon)}}\left\|r^{\gamma} \mid D^{2} u\right\|_{L_{2}\left(\Omega_{i}\right)}^{2} .
\end{aligned}
$$


Similarly from (3.23) we get

$$
\left\|r^{\beta}\left|D^{1}\left(u-v_{i}\right)\right|\right\|_{L_{2}\left(\Omega_{i}\right)}^{2} \leq C\left(\frac{d_{i}+C h_{i}}{d_{i}}\right)^{2(\beta+2)} \frac{h_{i}^{2}}{\left(d_{i}+C h_{i}\right)^{2 \varepsilon}}\left\|r^{\gamma}\left|D^{2} u\right|\right\|_{L_{2}\left(\Omega_{i}\right)}^{2} .
$$

Observe that there exists a constant $\bar{C}>1$ independent of $i$ but depending on the regularity of $\Delta$ such that $1<\frac{d_{i}+C h_{i}}{d_{i}} \leq \bar{C}$. Therefore estimates (3.25) and (3.26) yield

$$
\left\|u-v_{i}\right\|_{H_{\alpha, \beta}^{1}\left(\Omega_{i}\right)} \leq C h_{i}^{1-\varepsilon}\left\|r^{\gamma}\left|D^{2} u\right|\right\|_{L_{2}\left(\Omega_{i}\right)} \leq C h^{1-\varepsilon}\left\|r^{\gamma}\left|D^{2} u\right|\right\|_{L_{2}\left(\Omega_{i}\right)} .
$$

(ii) Case $\kappa>0$ (graded meshes). Recalling that $\gamma \leq \alpha+1+\varepsilon$ we deduce from (3.22) and (3.24) that

$$
\begin{aligned}
\left\|r^{\alpha}\left(u-v_{i}\right)\right\|_{L_{2}\left(\Omega_{i}\right)} & \leq C\left(\frac{h_{i}}{h}\right)^{\frac{\alpha}{\kappa}} h_{i}^{2}\left\|D^{2} u\right\|_{L_{2}\left(\Omega_{i}\right)} \leq C\left(\frac{h_{i}}{h}\right)^{\frac{-1-\varepsilon}{\kappa}} h_{i}^{2}\left\|r^{\gamma}\left|D^{2} u\right|\right\|_{L_{2}\left(\Omega_{i}\right)} \\
& =C\left(h_{i}^{1-\frac{\varepsilon}{\kappa}} h^{\frac{\varepsilon}{\kappa}}\right)\left(h_{i}^{1-\frac{1}{\kappa}} h^{\frac{1}{\kappa}}\right)\left\|r^{\gamma}\left|D^{2} u\right|\right\|_{L_{2}\left(\Omega_{i}\right)} \\
& \leq C \begin{cases}h\left\|r^{\gamma}\left|D^{2} u\right|\right\|_{L_{2}\left(\Omega_{i}\right)} & \text { if } \varepsilon \leq \kappa<1, \\
h^{\frac{1-\varepsilon}{1-\kappa}}\left\|r^{\gamma}\left|D^{2} u\right|\right\|_{L_{2}\left(\Omega_{i}\right)} & \text { if } 0<\kappa<\varepsilon .\end{cases}
\end{aligned}
$$

Using (3.23) and similar calculations we obtain

$$
\left\|r^{\beta}\left|D^{1}\left(u-v_{i}\right)\right|\right\|_{L_{2}\left(\Omega_{i}\right)} \leq C \begin{cases}h\left\|r^{\gamma}\left|D^{2} u\right|\right\|_{L_{2}\left(\Omega_{i}\right)} & \text { if } \varepsilon \leq \kappa<1, \\ h^{\frac{1-\varepsilon}{1-\kappa}}\left\|r^{\gamma}\left|D^{2} u\right|\right\|_{L_{2}\left(\Omega_{i}\right)} & \text { if } 0<\kappa<\varepsilon .\end{cases}
$$

Eventually we define $v \in S_{h}(\kappa, \sigma)$ by $v:=v_{0}$ on $\Omega_{0}$ and $v:=v_{i}$ on $\Omega_{i}$ for elements $\Omega_{i}$ separate from $O$. Then, combining estimates (3.21), (3.27) in the case $\kappa=0$ and combining (3.21), (3.28), (3.29) in the case $\kappa>0$, we obtain (2.7).

Now we analyze the regularity of problem (1.1), and prove Theorem 2.3. For simplicity we consider problem (1.1) with constant coefficients $a \equiv 1$ and $b \equiv 1$. The extension of our arguments and results to the general case is standard. We also note that the weak solution $u$ to problem (1.1) belongs to $H^{2}\left(\Omega \backslash \bar{B}_{\delta}\right)$ for any disk $B_{\delta} \subset \Omega$, because $\Omega$ is a convex polygon. Thus we conclude that in order to prove Theorem 2.3 one needs to study the regularity of the weak solution to the following problem:

$$
\begin{aligned}
\mathcal{L} u:=-\operatorname{div}\left(r^{2 \beta} \nabla u\right)+r^{2 \alpha} u & =f \quad \text { in } B_{\delta}, \\
u & =0 \text { on } \partial B_{\delta}
\end{aligned}
$$

(here, $B_{\delta} \subset \Omega$ denotes the disk of radius $\delta$ with center at the origin).

First, let us prove several auxiliary results. To simplify notation we shall write $\|\cdot\|$ for the norm $\|\cdot\|_{L_{2}\left(B_{\delta}\right)}$.

Lemma 3.10. Let $\alpha>-1, \beta \geq 0$, and $\alpha \geq \beta-1$. Assume that the function $u$ defined on $B_{\delta}$ satisfies $u=0$ on $\partial B_{\delta}$, and for some $\eta>-1$ there hold

$$
\left\|r^{\eta-1} u\right\|+\left\|r^{\eta}\left|D^{1} u\right|\right\|<+\infty \quad \text { and } \quad \mathcal{L} u \in L_{2, \eta+1-2 \beta}\left(B_{\delta}\right) .
$$

Then

$$
\left\|r^{\eta+1}\left|D^{2} u\right|\right\| \leq C\left(\|\mathcal{L} u\|_{L_{2, \eta+1-2 \beta}\left(B_{\delta}\right)}+\left\|r^{\eta-1} u\right\|+\left\|r^{\eta}\left|D^{1} u\right|\right\|\right) .
$$


Proof. For any $\eta_{1}, \eta_{2}$ one has

$$
r^{\eta_{1}} \operatorname{div}\left(r^{\eta_{2}} \nabla u\right)=\Delta\left(r^{\eta_{1}+\eta_{2}} u\right)-\left(\nabla r^{\eta_{1}+\eta_{2}}+r^{\eta_{2}} \nabla r^{\eta_{1}}\right) \cdot \nabla u-\Delta r^{\eta_{1}+\eta_{2}} u .
$$

Using this identity and simple calculations we find

$$
\begin{aligned}
r^{\eta+1-2 \beta} \mathcal{L} u=-\Delta\left(r^{\eta+1} u\right)+ & {\left[2(\eta+1-\beta) r^{\eta} \nabla u \cdot \nabla r\right.} \\
& \left.+\left((\eta+1)^{2} r^{\eta-1}+r^{\eta+1+2(\alpha-\beta)}\right) u\right] \\
=: \mathcal{L}_{0} u+\mathcal{L}_{1} u . &
\end{aligned}
$$

Applying the regularity of the Laplacian we have

$$
\begin{aligned}
\left\|\mathcal{L}_{0} u\right\|^{2} & =\left\|-\Delta\left(r^{\eta+1} u\right)\right\|^{2} \geq C\left|r^{\eta+1} u\right|_{H^{2}\left(B_{\delta}\right)}^{2} \\
& \geq C_{1}\left\|r^{\eta+1}\left|D^{2} u\right|\right\|^{2}-C_{2}\left(\left\|r^{\eta-1} u\right\|^{2}+\left\|r^{\eta}\left|D^{1} u\right|\right\|^{2}\right) .
\end{aligned}
$$

On the other hand, (3.32) yields

$$
\left\|\mathcal{L}_{0} u\right\|=\left\|r^{\eta+1-2 \beta} \mathcal{L} u-\mathcal{L}_{1} u\right\| \leq\|\mathcal{L} u\|_{L_{2, \eta+1-2 \beta}\left(B_{\delta}\right)}+C\left(\left\|r^{\eta-1} u\right\|+\left\|r^{\eta} \mid D^{1} u\right\| \|\right)
$$

(here we also used the assumption $\alpha \geq \beta-1$ ).

Then the statement of the lemma follows from inequalities (3.33) and (3.34).

Remark 3.2. Let $\alpha>-1, \beta=0$, and let $u \in \stackrel{\circ}{H}_{\alpha, 0}^{1}\left(B_{\delta}\right)$ be the weak solution to problem (3.30) for given $f \in L_{2, \mu}\left(B_{\delta}\right)$ with $\mu<1$. Then $u$ satisfies the assumptions of Lemma 3.10 for any $\eta>0$. In fact, by using Lemma 3.2 we check that

$$
\left\|r^{\eta-1} u\right\|+\left\|r^{\eta}\left|D^{1} u\right|\right\| \leq C\left\|r^{\eta}\left|D^{1} u\right|\right\| \leq C\|u\|_{H_{\alpha, 0}^{1}\left(B_{\delta}\right)},
$$

and $\mathcal{L} u=f \in L_{2, \mu}\left(B_{\delta}\right) \subset L_{2, \eta+1}\left(B_{\delta}\right)$, because $\mu<1$.

Thus by Lemma 3.10 we conclude that $u \in H_{\alpha, 0, \eta+1}^{2}\left(B_{\delta}\right)$ for any $\eta>0$. Now applying Lemma 3.5 we prove that for any small $\eta>0$ the function $r^{2 \eta} u=$ $r^{\eta+1-(1-\eta)} u$ is continuous on $\bar{B}_{\delta}$ and $\left(r^{2 \eta} u\right)(0,0)=0$.

Lemma 3.11. Let $\alpha>-1, \beta \geq 0$, and $\alpha \geq \beta-1$. Let $u \in \stackrel{\circ}{H}_{\alpha, \beta}^{1}\left(B_{\delta}\right)$ be the weak solution to problem (3.30) for given $f \in L_{2, \mu}\left(B_{\delta}\right)$. Then there exists $\lambda>0$ such that

$$
\left\|r^{\beta-\lambda-1} u\right\|+\left\|r^{\beta-\lambda}\left|D^{1} u\right|\right\| \leq C\|f\|_{L_{2, \mu}\left(B_{\delta}\right)},
$$

provided that $\mu \leq(1-\beta)-\lambda$.

Proof. We shall consider the cases $\beta>0$ and $\beta=0$ separately.

1) Let $\alpha+1 \geq \beta>0$. Instead of (3.30) we shall consider the problem

$$
\begin{aligned}
-\operatorname{div}\left(r^{2 \beta-2 \lambda} \nabla u\right)+r^{2 \beta} \nabla r^{-2 \lambda} \cdot \nabla u+r^{2 \alpha-2 \lambda} u & =r^{-2 \lambda} f \text { in } B_{\delta}, \\
u & =0 \text { on } \partial B_{\delta} .
\end{aligned}
$$

Observe that problem (3.30) is reduced to (3.36) by multiplying the differential equation in (3.30) by $r^{-2 \lambda}$ and performing elementary calculations. Let us consider the bilinear form corresponding to (3.36):

$$
A_{2}(u, v)=\int_{B_{\delta}}\left(r^{2(\beta-\lambda)} \nabla u \nabla v-2 \lambda r^{2(\beta-\lambda)-1} \nabla u \nabla r v+r^{2(\alpha-\lambda)} u v\right) d x .
$$


Its continuity is proved by using the Schwarz inequality and Lemma 3.2, provided that $\alpha-\lambda>-1$ and $\beta-\lambda>0$. To prove the $\stackrel{\circ}{H}_{\alpha-\lambda, \beta-\lambda}^{1}\left(B_{\delta}\right)$-ellipticity of $A_{2}(u, v)$ we write

$$
A_{2}(u, u)=\left\|r^{\beta-\lambda}\left|D^{1} u\right|\right\|^{2}-2 \lambda \int_{B_{\delta}} r^{2(\beta-\lambda)-1} \nabla u \nabla r u d x+\left\|r^{\alpha-\lambda} u\right\|^{2} .
$$

Applying Lemma 3.2 one has

$$
\left|2 \lambda \int_{B_{\delta}} r^{2(\beta-\lambda)-1} \nabla u \nabla r u d x\right| \leq 2 \lambda\left\|r^{\beta-\lambda}\left|D^{1} u\right|\right\|\left\|r^{\beta-\lambda-1} u\right\| \leq \frac{2 \lambda}{\beta-\lambda}\left\|r^{\beta-\lambda}\left|D^{1} u\right|\right\|^{2} .
$$

Then we obtain the desired property from (3.37):

$$
A_{2}(u, u) \geq\left(1-\frac{2 \lambda}{\beta-\lambda}\right)\|u\|_{H_{\alpha-\lambda, \beta-\lambda}^{1}\left(B_{\delta}\right)}^{2},
$$

provided $0<\lambda<\beta / 3$.

The linear functional $F_{2}(v)$ corresponding to problem 3.36 is continuous if $f \in L_{2, \mu}\left(B_{\delta}\right)$ with $\mu \leq(1-\beta)-\lambda$. In fact, for these values of $\mu$ and for any $v \in \stackrel{\circ}{H}_{\alpha-\lambda, \beta-\lambda}^{1}\left(B_{\delta}\right)$ one has

$\left|F_{2}(v)\right|=\left|\int_{B_{\delta}} r^{-2 \lambda} f v d x\right| \leq\left\|r^{-\lambda+1-\beta} f\right\|\left\|r^{\beta-\lambda-1} v\right\| \leq C\|f\|_{L_{2, \mu}\left(B_{\delta}\right)}\|v\|_{H_{\alpha-\lambda, \beta-\lambda}^{1}\left(B_{\delta}\right)}$.

Now for any $0<\lambda<\beta / 3$ the existence and uniqueness of the weak solution $u \in \stackrel{\circ}{H}_{\alpha-\lambda, \beta-\lambda}^{1}\left(B_{\delta}\right)$ to problem (3.36) is obtained by the Lax-Milgram lemma. Moreover

$$
\|u\|_{H_{\alpha-\lambda, \beta-\lambda}^{1}\left(B_{\delta}\right)} \leq C\|f\|_{L_{2, \mu}\left(B_{\delta}\right)} .
$$

Since $\alpha-\lambda>-1$ and $\beta-\lambda>0$, it remains to apply Lemma 3.2 once again, and inequality (3.35) follows for $\beta>0$.

2) Let $\alpha>-1$ and $\beta=0$ (note that $\alpha>\beta-1$ ). Then problem (3.30) reads

$$
\begin{aligned}
-\Delta u+r^{2 \alpha} u & =f \quad \text { in } B_{\delta}, \\
u & =0 \text { on } \partial B_{\delta} .
\end{aligned}
$$

For some $\rho \in(0, \delta / 2)$ we introduce a cut-off function $\chi(r)$ satisfying

$$
\left\{\begin{array}{l}
\chi(r)=0 \text { for } 0 \leq r \leq \rho, \quad \chi(r)=1 \text { for } r \geq 2 \rho \\
\chi \in C^{\infty}\left(\mathbb{R}_{+}\right), \quad 0 \leq \chi(r) \leq 1
\end{array}\right.
$$

Let us consider the function $v$ such that

$$
\nabla v=r^{-2 \lambda} \chi \nabla u \text { in } B_{\delta} \text { for } \lambda>0 .
$$

This function can be defined by solving

$$
\begin{aligned}
-\Delta v & =\tilde{f} \quad \text { in } B_{\delta}, \\
v & =0 \text { on } \partial B_{\delta},
\end{aligned}
$$

where $\tilde{f}=-\operatorname{div}\left(r^{-2 \lambda} \chi \nabla u\right)=-\chi \nabla r^{-2 \lambda} \cdot \nabla u-r^{-2 \lambda} \nabla \chi \cdot \nabla u-r^{-2 \lambda} \chi \Delta u$. 
Note that $\tilde{f} \in L_{2}\left(B_{\delta}\right)$, and problem (3.39) has a unique solution $v \in \stackrel{\circ}{H^{1}}\left(B_{\delta}\right) \cap$ $H^{2}\left(B_{\delta}\right)$. Taking $v$ as the test function in the integral identity for problem (3.38), using the Schwarz inequality and Lemma 3.2 we have

$$
\begin{aligned}
\int_{B_{\delta}} r^{-2 \lambda}|\nabla u|^{2} \chi d x & =\int_{B_{\delta}}|\nabla u \| \nabla v| d x=\int_{B_{\delta}}\left(f v-r^{2 \alpha} u v\right) d x \\
& \leq\left\|r^{1-\lambda} f\right\|\left\|r^{\lambda-1} v\right\|+\left\|r^{2 \alpha+1-\lambda} u\right\|\left\|r^{\lambda-1} v\right\| \\
& \leq C\left(\left\|r^{1-\lambda} f\right\|+\left\|r^{2 \alpha+2-\lambda}|\nabla u|\right\|\right)\left\|r^{\lambda}|\nabla v|\right\| \\
& \leq C\left(\|f\|_{L_{2, \mu}\left(B_{\delta}\right)}+\|u\|_{H_{\alpha, 0}^{1}\left(B_{\delta}\right)}\right)\left\|r^{-\lambda} \chi|\nabla u|\right\|,
\end{aligned}
$$

provided that $0<\lambda<2 \alpha+2$ and $\mu \leq 1-\lambda$.

Hence for any $\vartheta>0$ we find

$$
\int_{B_{\delta}} r^{-2 \lambda}|\nabla u|^{2} \chi d x \leq C \vartheta \int_{B_{\delta}} r^{-2 \lambda}|\nabla u|^{2} \chi d x+\frac{C}{\vartheta}\left(\|f\|_{L_{2, \mu}\left(B_{\delta}\right)}^{2}+\|u\|_{H_{\alpha, 0}^{1}\left(B_{\delta}\right)}^{2}\right) .
$$

Then taking $\vartheta>0$ sufficiently small and using the analogue of inequality (2.3) we obtain for any $\rho \in(0, \delta / 2)$

$$
\int_{B_{\delta} \backslash B_{2 \rho}} r^{-2 \lambda}|\nabla u|^{2} d x \leq \int_{B_{\delta}} r^{-2 \lambda}|\nabla u|^{2} \chi d x \leq C\|f\|_{L_{2, \mu}\left(B_{\delta}\right)}^{2}
$$

with constant $C>0$ independent of $\rho$. Therefore, letting $\rho \rightarrow 0$ we derive

$$
\left\|r^{-\lambda}\left|D^{1} u\right|\right\| \leq C\|f\|_{L_{2, \mu}\left(B_{\delta}\right)},
$$

provided that $0<\lambda<2 \alpha+2$ and $f \in L_{2, \mu}\left(B_{\delta}\right)$ with $\mu \leq 1-\lambda$.

It remains to show that for $\lambda>0$ there holds

$$
\left\|r^{-\lambda-1} u\right\| \leq C\left\|r^{-\lambda}\left|D^{1} u\right|\right\| .
$$

In fact, recalling Remark 3.2 and applying statement 1) of Lemma 3.1 we have for any $\tilde{\eta}>0$ :

$$
\begin{aligned}
\left\|r^{-\lambda-1} u\right\| & =\left\|r^{-\lambda-1-\tilde{\eta}}\left(r^{\tilde{\eta}} u\right)\right\| \\
& \leq C\left\|r^{-\lambda-\tilde{\eta}}\left|D^{1}\left(r^{\tilde{\eta}} u\right)\right|\right\| \leq C \tilde{\eta}\left\|r^{-\lambda-1} u\right\|+C\left\|r^{-\lambda}\left|D^{1} u\right|\right\| .
\end{aligned}
$$

Now taking $\tilde{\eta}>0$ small enough we obtain (3.41). Then inequality (3.35) for $\beta=0$ follows from (3.40) and (3.41).

Proof of Theorem 2.3. As mentioned above one needs to study the regularity of the weak solution to problem (3.30). By Lemma 3.11 there exists $\lambda>0$ such that inequality (3.35) holds if $f=\mathcal{L} u \in L_{2, \mu}\left(B_{\delta}\right) \subset L_{2,1-\beta-\lambda}\left(B_{\delta}\right)$. Thus, for given $f \in L_{2, \mu}\left(B_{\delta}\right)$ with $\mu \leq 1-\beta-\lambda$, all assumptions of Lemma 3.10 are satisfied with $\eta:=\beta-\lambda$, and therefore

$$
\left\|r^{\beta-\lambda+1}\left|D^{2} u\right|\right\| \leq C\|f\|_{L_{2, \mu}\left(B_{\delta}\right)}, \quad \mu \leq 1-\beta-\lambda .
$$

Now taking $\lambda:=1-\varepsilon$ for some $\varepsilon \in[0,1)$ we obtain the assertion of the theorem. 
Proof of Theorem 2.4. Let $\Omega_{1}$ be an element touching the singularity point $O$, let $R_{\tilde{h}}=\left\{(r, \theta) \mid 0<r<\tilde{h}, 0<\theta<\theta_{0}\right\} \subset \Omega_{1}, \tilde{h}=h / 2$, and let $u_{h}(x)=a x_{1}+b x_{2}+c$ for $x \in \Omega_{1}$. Then for $h$ sufficiently small (such that $\operatorname{diam}\left(\Omega_{1}\right)<\rho$; note that $\rho$ was used to define the cut-off function $\chi$ in (2.10) ) one has

$$
\begin{aligned}
\left\|u_{\text {model }}-u_{h}\right\|_{H_{\alpha, \beta}^{1}(\Omega)}^{2} & \geq\left\|r^{\beta} \mid D^{1}\left(u_{\text {model }}-u_{h}\right)\right\|_{L_{2}\left(R_{\tilde{h}}\right)}^{2} \\
& \geq \int_{0}^{\theta_{0}} \int_{0}^{\tilde{h}} r^{2 \beta}\left(\lambda r^{\lambda-1} \cos \theta-a\right)^{2} r d r d \theta \\
& =\frac{\lambda^{2} \tilde{h}^{2 \beta+2 \lambda}}{2 \beta+2 \lambda} \int_{0}^{\theta_{0}} \cos ^{2} \theta d \theta \\
& +\left[a^{2} \theta_{0} \frac{\tilde{h}^{2 \beta+2}}{2 \beta+2}-2 \lambda a \sin \theta_{0} \frac{\tilde{h}^{2 \beta+\lambda+1}}{2 \beta+\lambda+1}\right] \\
& \geq \frac{\lambda^{2} \tilde{h}^{2 \beta+2 \lambda}\left(\cos \theta_{0} \sin \theta_{0}+\theta_{0}\right)}{4(\beta+\lambda)}-\frac{\sin ^{2} \theta_{0}(2 \beta+2)}{(2 \beta+\lambda+1)^{2} \theta_{0}} \lambda^{2} \tilde{h}^{2 \beta+2 \lambda} \\
& =\frac{\lambda^{2} C_{1}\left(\theta_{0}\right)}{C_{2}\left(\theta_{0}\right)} h^{2(\beta+\lambda)},
\end{aligned}
$$

where

$$
\begin{aligned}
& C_{1}\left(\theta_{0}\right)=((\beta+\lambda)+(\beta+1))^{2}\left(\sin 2 \theta_{0}+2 \theta_{0}\right) \theta_{0}-16 \sin ^{2} \theta_{0}(\beta+\lambda)(\beta+1), \\
& C_{2}\left(\theta_{0}\right)=2^{2(\beta+\lambda)+3} \theta_{0}(\beta+\lambda)(2 \beta+\lambda+1)^{2}>0 .
\end{aligned}
$$

Considering $C_{1}\left(\theta_{0}\right)$ as a quadratic function of $(\beta+\lambda)$ it is easy to show that $C_{1}\left(\theta_{0}\right)>$ 0 for any $\theta_{0}>0$. This finishes the proof.

Proof of Theorem 2.5. We shall follow the guidelines of the proof of Lemma 4.6 in [2]. Let $u \in S_{h}(\varepsilon, \sigma)$. We need to show that for any $t>0$ and $\theta \in(0,1 / 2]$

$$
K(t, u)=\inf _{u=v+w}\left(\|v\|_{H_{\alpha, \beta}^{1}(\Omega)}+t\|w\|_{H_{\alpha, \beta, \beta+\varepsilon}^{2}(\Omega)}\right) \leq C t^{\theta} h^{-\theta}\|u\|_{H_{\alpha, \beta}^{1}(\Omega)} .
$$

Setting $v:=u, w:=0$ we have for $t \geq h / 4$

$$
K(t, u) \leq\|u\|_{H_{\alpha, \beta}^{1}(\Omega)} \leq C t^{\theta} h^{-\theta}\|u\|_{H_{\alpha, \beta}^{1}(\Omega)} .
$$

Further, for any $\Omega_{i} \in \Delta(h, \varepsilon, \sigma)$ we denote $N\left(\Omega_{i}\right)=\bigcup\left\{\bar{\Omega}_{k} ; \Omega_{k} \in \Delta(h, \varepsilon, \sigma), \bar{\Omega}_{k} \cap\right.$ $\left.\bar{\Omega}_{i} \neq \varnothing\right\}$. In order to estimate $K(t, u)$ for $t \leq h / 4$ we use the function $w \in \stackrel{\circ}{H}^{1}(\Omega)$ which was explicitly constructed in the proof of Lemma 4.6 in [2]. One has $w \in$ $C^{2}(\bar{\Omega})$, and for any $\Omega_{i} \in \Delta(h, \varepsilon, \sigma)$ there holds

$$
\begin{gathered}
\sup _{x \in \Omega_{i}}\left|D^{1} w(x)\right| \leq C \sup _{x \in N\left(\Omega_{i}\right)}\left[|u(x)|+\left|D^{1} u(x)\right|\right], \\
\sup _{x \in \Omega_{i}}\left|D^{2} w(x)\right| \leq C \delta_{i}^{-1} \sup _{x \in N\left(\Omega_{i}\right)}\left[|u(x)|+\left|D^{1} u(x)\right|\right], \\
\operatorname{meas}\left\{x \in \bar{\Omega}_{i} ; u(x) \neq w(x)\right\} \leq C h_{i} \delta_{i},
\end{gathered}
$$

with $\delta_{i}=h^{-1} t h_{i}$. 
Using these properties we obtain

$$
\begin{gathered}
\left\|D^{1}(u-w)\right\|_{L_{2}\left(\Omega_{i}\right)}^{2} \leq C h_{i} \delta_{i} \sup _{x \in N\left(\Omega_{i}\right)} \sum_{l=0}^{1}\left|D^{l} u(x)\right|^{2} \leq C h_{i}^{-1} \delta_{i} \sum_{l=0}^{1}\left\|D^{l} u\right\|_{L_{2}\left(N\left(\Omega_{i}\right)\right)}^{2}, \\
\left\|D^{2} w\right\|_{L_{2}\left(\Omega_{i}\right)}^{2} \leq C h_{i}^{-1} \delta_{i}^{-1}\left(\|u\|_{L_{2}\left(N\left(\Omega_{i}\right)\right)}^{2}+\left\|D^{1} u\right\|_{L_{2}\left(N\left(\Omega_{i}\right)\right)}^{2}\right) .
\end{gathered}
$$

On the other hand, for any element $\Omega_{i} \in \Delta(h, \varepsilon, \sigma)$ and $\eta \geq 0$ there holds

$$
C h^{\frac{2 \eta}{1-\varepsilon}}\|v\|_{L_{2}\left(\Omega_{i}\right)}^{2} \leq\left\|r^{\eta} v\right\|_{L_{2}\left(\Omega_{i}\right)}^{2} \quad \text { if } O \in \bar{\Omega}_{i}
$$

for any linear function $v$, and

$$
\begin{gathered}
C\left(\frac{h_{i}}{h}\right)^{\frac{2 \eta}{\varepsilon}}\|v\|_{L_{2}\left(\Omega_{i}\right)}^{2} \leq\left\|r^{\eta} v\right\|_{L_{2}\left(\Omega_{i}\right)}^{2} \leq C\left(\frac{h_{i}}{h}\right)^{\frac{2 \eta}{\varepsilon}}\|v\|_{L_{2}\left(\Omega_{i}\right)}^{2} \quad \text { if } O \notin \bar{\Omega}_{i}, \quad \varepsilon \neq 0, \\
d_{i}^{2 \eta}\|v\|_{L_{2}\left(\Omega_{i}\right)}^{2} \leq\left\|r^{\eta} v\right\|_{L_{2}\left(\Omega_{i}\right)}^{2} \leq\left(d_{i}+C h_{i}\right)^{2 \eta}\|v\|_{L_{2}\left(\Omega_{i}\right)}^{2} \quad \text { if } O \notin \bar{\Omega}_{i}, \quad \varepsilon=0,
\end{gathered}
$$

for any $v \in L_{2}\left(\Omega_{i}\right)$. In fact, (3.45) follows by using scaling arguments, equivalence of norms on finite-dimensional spaces and inequalities (3.19), (3.20); (3.46) follows by scaling arguments and (3.19), (3.20); and (3.47) (respectively, (3.48) is a direct consequence of the definition of the graded (respectively, quasi-uniform) meshes. Then applying estimates (3.43), (3.44) together with (3.45)-(3.48) we obtain for any $u \in S_{h}(\varepsilon, \sigma)$

$$
\begin{gathered}
\left\|r^{\beta}\left|D^{1}(u-w)\right|\right\|_{L_{2}\left(\Omega_{i}\right)}^{2} \leq C h_{i}^{-1} \delta_{i}\|u\|_{H_{\beta, \beta}^{1}\left(N\left(\Omega_{i}\right)\right)}^{2} \quad \forall \Omega_{i} \in \Delta(h, \varepsilon, \sigma), \\
\left\|r^{\beta+\varepsilon}\left|D^{2} w\right|\right\|_{L_{2}\left(\Omega_{j}\right)}^{2} \leq C h^{\frac{2 \varepsilon}{1-\varepsilon}} h_{j}^{-1} \delta_{j}^{-1}\|u\|_{H_{\beta, \beta}^{1}\left(N\left(\Omega_{j}\right)\right)}^{2} \leq C h^{-2} \frac{h_{j}}{\delta_{j}}\|u\|_{H_{\beta, \beta}^{1}\left(N\left(\Omega_{j}\right)\right)}^{2}
\end{gathered}
$$

if $O \in \bar{\Omega}_{j}, \varepsilon \in[0,1)$

$$
\left\|r^{\beta+\varepsilon}\left|D^{2} w\right|\right\|_{L_{2}\left(\Omega_{i}\right)}^{2} \leq C\left(\frac{h_{i}}{h}\right)^{2} h_{i}^{-1} \delta_{i}^{-1}\|u\|_{H_{\beta, \beta}^{1}\left(N\left(\Omega_{i}\right)\right)}^{2}=C h^{-2} \frac{h_{i}}{\delta_{i}}\|u\|_{H_{\beta, \beta}^{1}\left(N\left(\Omega_{i}\right)\right)}^{2}
$$

if $O \notin \bar{\Omega}_{i}, \varepsilon \neq 0$, and

$$
\left\|r^{\beta}\left|D^{2} w\right|\right\|_{L_{2}\left(\Omega_{i}\right)}^{2} \leq C h_{i}^{-1} \delta_{i}^{-1}\|u\|_{H_{\beta, \beta}^{1}\left(N\left(\Omega_{i}\right)\right)}^{2} \leq C h^{-2} h_{i} \delta_{i}^{-1}\|u\|_{H_{\beta, \beta}^{1}\left(N\left(\Omega_{i}\right)\right)}^{2}
$$

if $O \notin \bar{\Omega}_{i}, \varepsilon=0$. Combining the above inequalities over all elements of the mesh, recalling that $\delta_{i}=h^{-1} t h_{i}, \alpha+1 \geq \beta$, and using Lemma 3.2 we derive

$$
\begin{gathered}
\|u-w\|_{H_{\alpha, \beta}^{1}(\Omega)} \leq C\left\|r^{\beta} \mid D^{1}(u-w)\right\|\left\|_{L_{2}(\Omega)} \leq C t^{1 / 2} h^{-1 / 2}\right\| u \|_{H_{\beta, \beta}^{1}(\Omega)} \\
\leq C t^{1 / 2} h^{-1 / 2}\|u\|_{H_{\alpha, \beta}^{1}(\Omega)}, \\
\|w\|_{H_{\alpha, \beta, \beta+\varepsilon}^{2}(\Omega)} \leq C t^{-1 / 2} h^{-1 / 2}\|u\|_{H_{\alpha, \beta}^{1}(\Omega)} .
\end{gathered}
$$

Hence inequality (3.42) follows for $t \in(0, h / 4]$ and for any $\theta \in(0,1 / 2]$.

Proof of Theorem 2.6. It suffices to prove that $f_{1} \in L_{2, \mu_{1}}(\Omega)$ for some $\mu_{1}<1$. In fact, let $\mu_{1}=\mu+2 \beta<1$. Then

$$
\left\|f_{1}\right\|_{L_{2, \mu_{1}}(\Omega)}=\left\|f a^{-1}\right\|_{L_{2, \mu}(\Omega)} \leq \underline{a}^{-1}\|f\|_{L_{2, \mu}(\Omega)}<\infty,
$$

and the required statement follows from Theorem 2.1. 
The results of Theorems 2.7 and 2.10 follow from Lemma 3.9 and Cea's lemma in the same way as in the proof of Theorem 2.2. Theorem 2.8 is an immediate corollary of Theorems 2.3 and 2.6 .

Proof of Theorem 2.9. Since $0<b(x) \leq \bar{b}$, and $\left|\frac{\partial r}{\partial x_{i}}\right| \leq 1(i=1,2)$, we have by the Schwarz inequality

$$
\begin{aligned}
& \left|A_{\varepsilon}(u, v)\right| \leq\left\|r^{\varepsilon}\left|D^{1} u\right|\right\|_{L_{2}(\Omega)}\left\|r^{\varepsilon}\left|D^{1} v\right|\right\|_{L_{2}(\Omega)} \\
& \quad+\left|\int_{\Omega} \nabla\left(r^{2 \varepsilon}\right) \nabla u v d x\right|+\bar{b}\left\|r^{\varepsilon-1} u\right\|_{L_{2}(\Omega)}\left\|r^{\varepsilon-1} v\right\|_{L_{2}(\Omega)},
\end{aligned}
$$

and

$$
\begin{aligned}
\left|\int_{\Omega} \nabla\left(r^{2 \varepsilon}\right) \nabla u v d x\right| & \leq 2 \varepsilon \sum_{i=1}^{2}\left\|r^{\varepsilon}\left|\frac{\partial u}{\partial x_{i}}\right|\right\|_{L_{2}(\Omega)}\left\|r^{\varepsilon-1} v\right\|_{L_{2}(\Omega)} \\
& \leq 2 \varepsilon \sqrt{2}\left\|r^{\varepsilon}\left|D^{1} u\right|\right\|_{L_{2}(\Omega)}\left\|r^{\varepsilon-1} v\right\|_{L_{2}(\Omega)} .
\end{aligned}
$$

Hence, for all $u, v \in \stackrel{\circ}{H}_{\varepsilon-1, \varepsilon}^{1}(\Omega)$,

$$
\left|A_{\varepsilon}(u, v)\right| \leq C\|u\|_{H_{\varepsilon-1, \varepsilon}^{1}(\Omega)}\|v\|_{H_{\varepsilon-1, \varepsilon}^{1}(\Omega)} .
$$

On the other hand inequality (3.49) yields

$$
\int_{\Omega} \nabla\left(r^{2 \varepsilon}\right) \nabla u v d x \geq-\varepsilon \sqrt{2}\left(\delta\left\|r^{\varepsilon}\left|D^{1} u\right|\right\|_{L_{2}(\Omega)}^{2}+\delta^{-1}\left\|r^{\varepsilon-1} v\right\|_{L_{2}(\Omega)}^{2}\right)
$$

with arbitrary $\delta>0$. Therefore, recalling that $b(x) \geq \underline{b}>0$ and $\varepsilon \in(0, \sqrt{2 \underline{b}} / 2)$, we choose a $\delta \in\left(\frac{\varepsilon \sqrt{2}}{\underline{b}}, \frac{1}{\varepsilon \sqrt{2}}\right)$ to obtain

$$
\begin{aligned}
A_{\varepsilon}(u, u) & =\int_{\Omega}\left(r^{2 \varepsilon}|\nabla u|^{2}+\nabla\left(r^{2 \varepsilon}\right) \nabla u u+b r^{2(\varepsilon-1)} u^{2}\right) d x \\
& \geq \min \left\{1-\varepsilon \delta \sqrt{2}, \underline{b}-\varepsilon \delta^{-1} \sqrt{2}\right\}\|u\|_{H_{\varepsilon-1, \varepsilon}^{1}(\Omega)}^{2} .
\end{aligned}
$$

Since $f \in L_{2,1+\varepsilon}(\Omega)$, we prove the continuity of $F_{\varepsilon}(v)$ by the Schwarz inequality:

$$
\left|F_{\varepsilon}(v)\right| \leq \int_{\Omega} r^{2 \varepsilon}|f||v| d x \leq\|f\|_{L_{2, \varepsilon+1}(\Omega)}\|v\|_{H_{\varepsilon-1, \varepsilon}^{1}(\Omega)} .
$$

Thus, $A_{\varepsilon}(u, v)$ and $F_{\varepsilon}(v)$ satisfy the assumptions of the Lax-Milgram lemma with respect to the space $\stackrel{\circ}{H}_{\varepsilon-1, \varepsilon}^{1}(\Omega)$, and the statement of the theorem follows.

Remark 3.3. If $\Omega \subset \mathbb{R}^{3}$ is a convex polyhedral domain, then similar to the twodimensional case one can introduce the weighted space $H_{\eta_{0}, \eta_{1}, \ldots, \eta_{k}}^{k}(\Omega)$ with real $\eta_{l}>-3 / 2(l=0,1, \ldots, k)$. Then several results of this section remain valid, however, with different restrictions for parameters. In particular, Lemma 3.1 is valid for $\alpha>-3 / 2$ and $\beta \neq-1 / 2$, Lemma 3.2 is true for $\beta>-1 / 2$, and Lemmas 3.3, 3.4 are satisfied for $\alpha, \beta>-3 / 2$ and for $-3 / 2<\gamma<1 / 2$. Therefore, considering problem (1.1) in $\Omega \subset \mathbb{R}^{3}$ with $\alpha, \beta>-3 / 2$, one can introduce the variational formulation analogous to (2.2) and prove its unique solvability. On the other hand, the results regarding the convergence of finite element approximations follow from 
the interpolation theory relying on the compactness property (see Lemma 3.6) and the estimate in Lemma 3.8. Lemma 3.6 and its corollary Lemma 3.7 are valid in $\mathbb{R}^{3}$ for $\alpha>-3 / 2, \beta>-1$, and $-1 / 2 \leq \gamma<\min \{\alpha+1, \beta\}+1 / 2$. However, the proof of Lemma 3.8 is not immediate in three dimensions. To overcome discontinuities in the approximations in the neighborhood of the singularity point (this is the central point in the proof of Lemma 3.9 one should use a different technique. For example, Clément's interpolation may work, but to our knowledge this is an open problem in weighted spaces.

\section{Numerical Results}

In this section we present numerical results which confirm the a priori error estimates formulated in $\$ 2.2$ For simplicity we use only uniform meshes. We consider two types of model problems (cf. (2.13), (2.15)):

$$
\text { Problem A. } \begin{aligned}
-\Delta u+r^{-1} u & =f \quad \text { in } \Omega, \\
u & =0 \text { on } \partial \Omega,
\end{aligned}
$$

and

$$
\text { Problem B. } \begin{aligned}
-\Delta u+r^{-2} u & =g \text { in } \Omega, \\
u & =0 \text { on } \partial \Omega .
\end{aligned}
$$

The weak formulations of problems (4.1) and (4.2) are given by (2.14) with $\alpha=$ $-\frac{1}{2}, \beta=0$ and (2.16) with some $\varepsilon \in\left(0, \frac{\sqrt{2}}{2}\right)$, respectively.

In our numerical examples we choose $\Omega=(-1,1) \times(-1,1)$ and take the righthand side functions $f, g$ in (4.1), (4.2) such that

$$
u\left(x_{1}, x_{2}\right)=\left(1-x_{1}^{2}\right)\left(1-x_{2}^{2}\right) r^{\eta}\left(x_{1}, x_{2}\right) \in V,
$$

where $V=\stackrel{\circ}{H_{-\frac{1}{2}, 0}^{1}}(\Omega)$ for Problem A, and $V=\stackrel{\circ}{H_{\varepsilon-1, \varepsilon}^{1}}(\Omega)$ for Problem B.

For the corresponding finite element schemes we use uniform triangulations of the domain and standard piecewise linear basis functions.

Observe that the lower coefficients in (4.1), (4.2) become infinite at the origin, and singularities may also appear in the right-hand side functions $f, g$ as well as in solution (4.3). Therefore, we pay special attention to accurate numerical integration over the elements in the neighborhood of the origin. The contributions of these elements to the stiffness matrix and to the right-hand side vector of the linear system are computed using the nonlinear Duffy transformation:

$$
M:\left\{\begin{array}{l}
x_{1}=\xi_{1}\left(c_{1}+\left(d_{1}-c_{1}\right) \xi_{2}\right), \\
x_{2}=\xi_{1}\left(c_{2}+\left(d_{2}-c_{2}\right) \xi_{2}\right),
\end{array}\right.
$$

where $\left(\xi_{1}, \xi_{2}\right) \in Q=[0,1] \times[0,1],\left(x_{1}, x_{2}\right) \in \bar{\Omega}_{i}$, and $\bar{\Omega}_{i}$ is an element of the mesh with the vertices $O(0,0), C\left(c_{1}, c_{2}\right)$, and $D\left(d_{1}, d_{2}\right)$. For the Jacobian of this transformation one has

$$
J=\xi_{1}\left(c_{1} d_{2}-c_{2} d_{1}\right),
$$

and for the weight function $r\left(x_{1}, x_{2}\right)$ we derive

$$
r\left(x_{1}, x_{2}\right)=\left(x_{1}^{2}+x_{2}^{2}\right)^{1 / 2}=\xi_{1}\left[\left(c_{1}+\left(d_{1}-c_{1}\right) \xi_{2}\right)^{2}+\left(c_{2}+\left(d_{2}-c_{2}\right) \xi_{2}\right)^{2}\right]^{1 / 2} .
$$

Thus transformation (4.4) allows us to separate variables in the integrands having singularities. Then the integration of the singular part with respect to $\xi_{1}$ is 
TABLE 1. The convergence of the method for the problems in Examples A1 and A2.

\begin{tabular}{c|r|c|c|c|c}
\multirow{2}{*}{\multicolumn{1}{c|}{}} & \multicolumn{2}{|c|}{ Example A1 } & \multicolumn{2}{c}{ Example A2 } \\
\cline { 3 - 6 } & \multicolumn{1}{c}{$N$} & $E_{h}$ & Convergence rate & $E_{h}$ & Convergence rate \\
\hline 0.500000 & 9 & 0.488279 & - & 0.627086 & - \\
0.250000 & 49 & 0.277692 & 0.814 & 0.341157 & 0.878 \\
0.125000 & 225 & 0.164437 & 0.755 & 0.176095 & 0.954 \\
0.062500 & 961 & 0.102674 & 0.679 & 0.089463 & 0.976 \\
0.041667 & 2209 & 0.079739 & 0.623 & 0.060021 & 0.984 \\
0.031250 & 3969 & 0.067206 & 0.594 & 0.045183 & 0.987 \\
0.025000 & 6241 & 0.059093 & 0.576 & 0.036238 & 0.988 \\
0.020833 & 9025 & 0.053316 & 0.564 & 0.030255 & 0.989 \\
0.017857 & 12321 & 0.048940 & 0.555 & 0.025971 & 0.990 \\
0.015625 & 16129 & 0.045482 & 0.548 & 0.022752 & 0.991
\end{tabular}

performed analytically. The remaining regular part (a smooth function of $\xi_{2}$ ) is integrated numerically via the Romberg quadrature rule (see [20]).

In the numerical examples below we choose different values of the parameter $\eta$ in (4.3) to show how the method can treat various types of singularities. In each case the approximate solutions $u_{h}$ are computed on a sequence of triangulations and the corresponding relative errors $E_{h}=\frac{\left\|u-u_{h}\right\|_{V}}{\|u\|_{V}}$ are calculated. Using a double logarithmic scale we plot $E_{h}$ as a function of the dimension $N$ of the finite element space and compare the rate of convergence with the rate predicted by Theorems 2.7 and 2.10.

For Problem A we consider $\eta=0.5$ (Example A1) and $\eta=1.2$ (Example A2). In the first case the solution has a weak singularity at the origin, because $u \in H^{1}(\Omega)$, but $u \notin H^{2}(\Omega)$. Observing that $u \in H_{-\frac{1}{2}, 0, \tilde{\varepsilon}}^{2}(\Omega)$ for any $\tilde{\varepsilon}>0.5$, we conclude by Theorem 2.7 that the rate of convergence should be close to $O\left(h^{0.5}\right)$. The results of the numerical computations for this case are given in Table 1, and Figure 3 shows the decay of the error $E_{h}(N)$ in comparison with the theoretically predicted rate of convergence.

In Example A2 the solution to problem (4.1) is sufficiently smooth, $u \in H^{2}(\Omega)$. Therefore by Theorem 2.7 we expect the rate of convergence $O(h)$, which is confirmed by the computations (see Table 1 and Figure 4 ).

The following three examples correspond to Problem B.

Example B1: $\eta=-0.2$. In this case we have a strongly singular solution. Indeed, $u$ is infinite at the origin and $u \notin H^{1}(\Omega)$. Taking $\varepsilon=0.65$ in (2.16), we see that $u \in H_{\varepsilon-1, \varepsilon, \varepsilon+\tilde{\varepsilon}}^{2}(\Omega)$ for any $\tilde{\varepsilon}>0.55$. Hence the rate of convergence predicted by Theorem 2.10 should be close to $O\left(h^{1-\tilde{\varepsilon}}\right)=O\left(h^{0.45}\right)$. The numerical results are presented in Table 2 and Figure 5 . As in Example A1 we observe that the error of approximation $E_{h}$ decays with the growth of $N$, and the rate of convergence approaches the rate predicted by our theory.

Example B2: $\eta=0.5$. As in Example A1, here we consider the case of a weakly singular solution. Taking $\varepsilon=0.6$ in (2.16), one has $u \in H_{\varepsilon-1, \varepsilon, \varepsilon}^{2}(\Omega)$, and Theorem 2.10 predicts that the error $E_{h}$ decays like $O(h)$. 


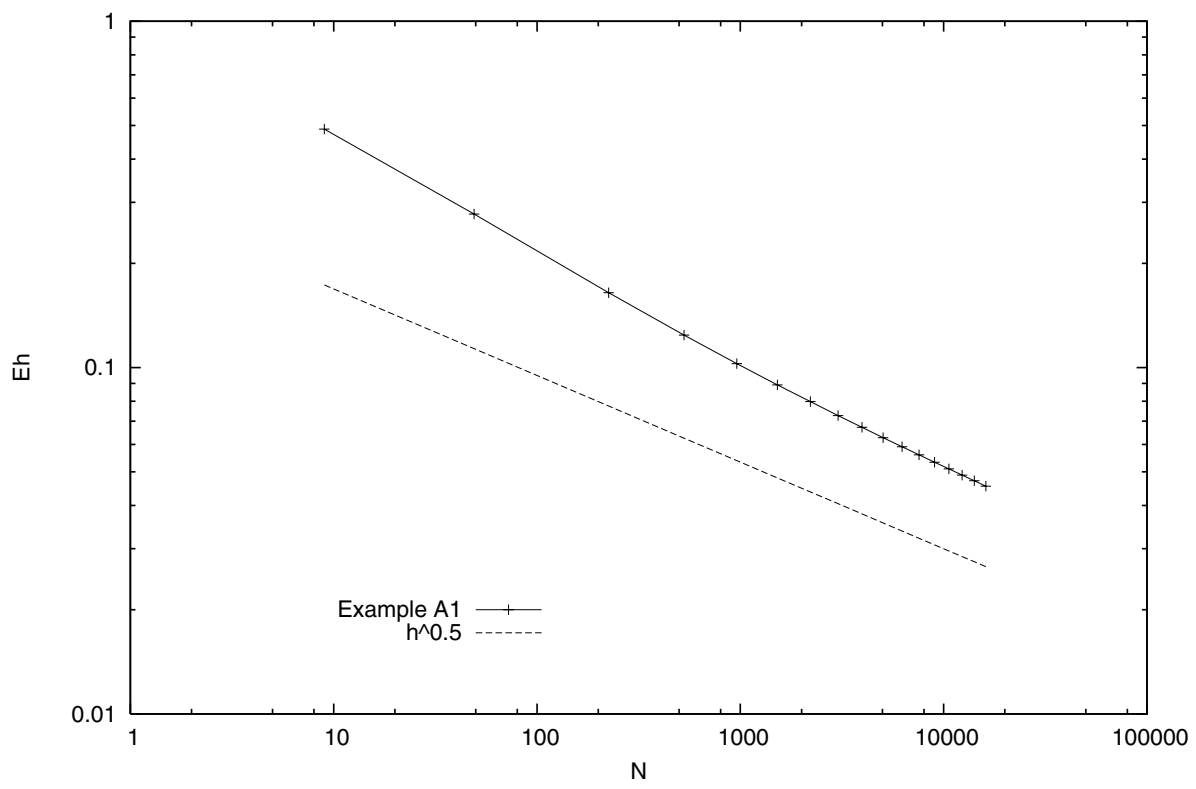

Figure 3 . The errors of approximation $E_{h}$ for the problem in Example A1.

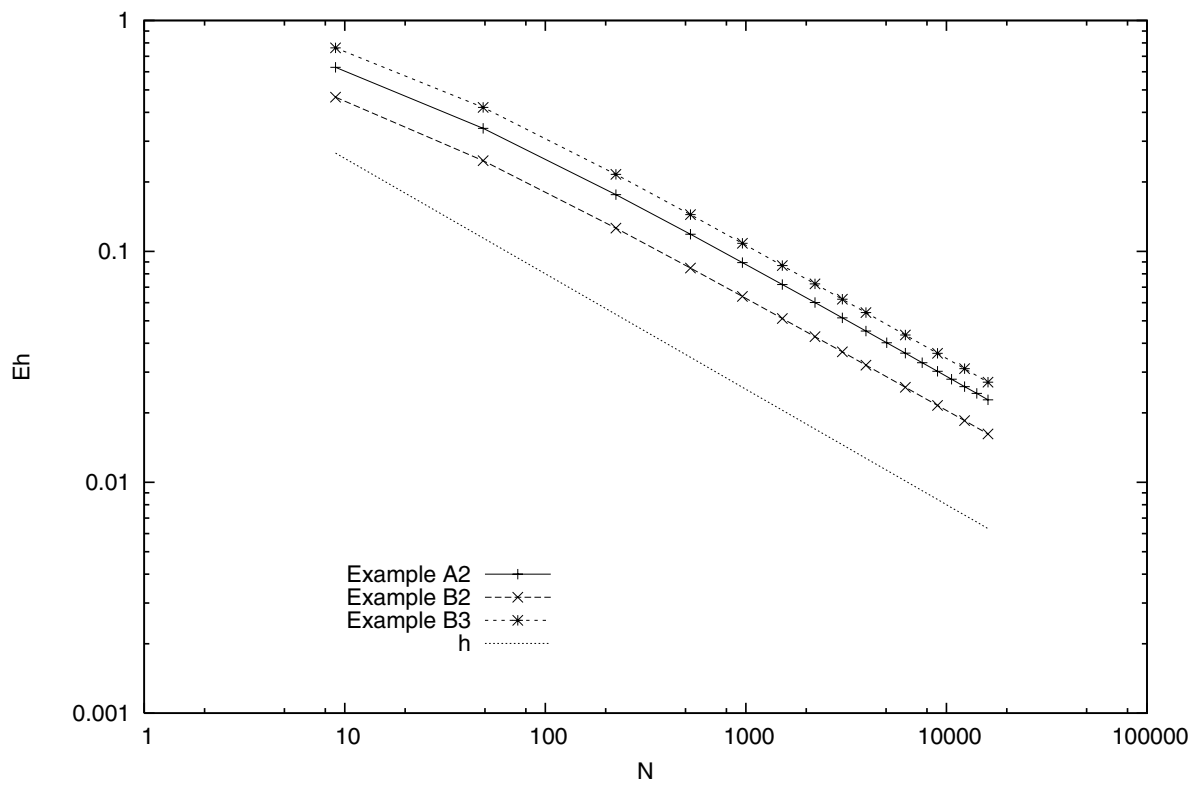

FiguRE 4. The errors of approximation $E_{h}$ for the problems in Examples A2, B2, and B3. 
TABLE 2. The convergence of the method for the problems in Examples B1, B2, B3.

\begin{tabular}{c|r|r|c|c|c|c|c}
\multirow{2}{*}{\multicolumn{1}{c|}{}} & \multicolumn{2}{|c|}{ Example B1 } & \multicolumn{2}{c|}{ Example B2 } & \multicolumn{2}{c}{ Example B3 } \\
\cline { 3 - 8 } & \multicolumn{1}{c|}{$N$} & $E_{h}$ & Conv. rate & $E_{h}$ & Conv. rate & $E_{h}$ & Conv. rate \\
\hline 0.500000 & 9 & 0.327870 & - & 0.465402 & - & 0.761223 & - \\
0.250000 & 49 & 0.184586 & 0.828 & 0.246925 & 0.914 & 0.420598 & 0.855 \\
0.125000 & 225 & 0.109700 & 0.750 & 0.126183 & 0.968 & 0.215512 & 0.964 \\
0.062500 & 961 & 0.069725 & 0.653 & 0.063817 & 0.983 & 0.108415 & 0.991 \\
0.041667 & 2209 & 0.055008 & 0.584 & 0.042759 & 0.987 & 0.072358 & 0.997 \\
0.031250 & 3969 & 0.046961 & 0.549 & 0.032171 & 0.989 & 0.054290 & 0.998 \\
0.025000 & 6241 & 0.041734 & 0.528 & 0.025795 & 0.989 & 0.043440 & 0.999 \\
0.020833 & 9025 & 0.037994 & 0.514 & 0.021534 & 0.990 & 0.036204 & 0.999 \\
0.017857 & 12321 & 0.035148 & 0.505 & 0.018484 & 0.990 & 0.031033 & 0.999 \\
0.015625 & 16129 & 0.032888 & 0.497 & 0.016193 & 0.991 & 0.027155 & 0.999
\end{tabular}

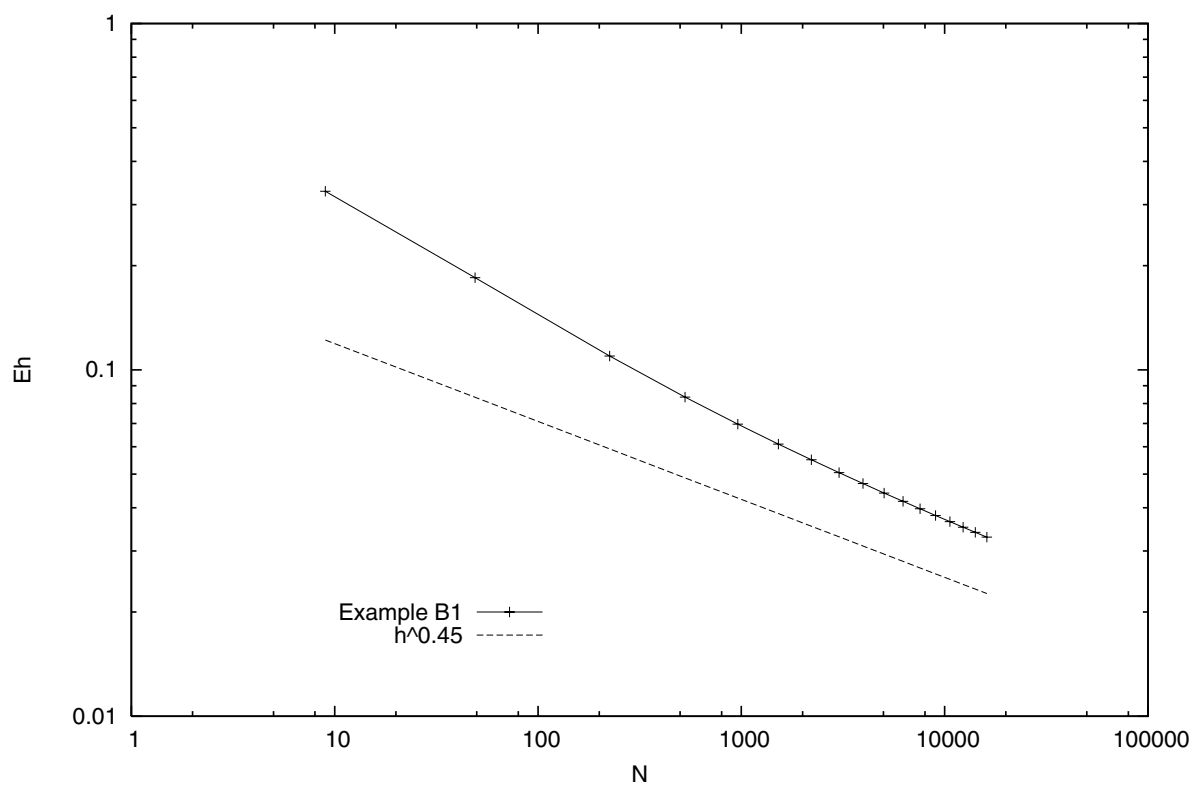

Figure 5. The errors of approximation $E_{h}$ for the problem in Example B1.

Example B3: $\eta=2$. In this example one has a smooth solution. We take $\varepsilon=0.05$ in (2.16), and obtain $u \in H_{\varepsilon-1, \varepsilon, \varepsilon}^{2}(\Omega)$. Thus by Theorem 2.10 we expect the rate of convergence to be $O(h)$ in this case.

The results of computations for the problems in Examples B2, B3 are given in Table 2, and are also shown in Figure 4. We see that in both these examples the errors $E_{h}$ decay like $O(h)$.

Thus our numerical experiments confirm the theoretical error bounds given in \$2.2. The results of Example B3 also show the robustness of the method for sufficiently small $\varepsilon$. On the other hand, the lack of the regularity of the solutions in 
Examples A1 and B1 does not allow us to achieve the rate of convergence $O(h)$ (this fact is also predicted by Theorems 2.7 and 2.10).

\section{ACKNOWLEDGMENTS}

Main parts of this work were accomplished while A. Bespalov was visiting N. Heuer as a postdoctoral researcher at the Departamento de Ingeniería Matemática of the Universidad de Concepción, Chile. A. Bespalov wishes to express his gratitude to the colleagues of that department for their hospitality and the stimulating research atmosphere.

\section{REFERENCES}

[1] I. BABUŠKA, Error bounds for the finite element method, Numer. Math., 16 (1971), pp. $322-$ 333. MR0288971(44:6166)

[2] I. Babuška, R. B. KellogG, and J. PitkÄranta, Direct and inverse error estimates for finite elements with mesh refinement, Numer. Math., 33 (1979), pp. 447-471. MR 0553353 (81c:65054)

[3] J. Bergh and J. LÖFström, Interpolation Spaces, no. 223 in Grundlehren der mathematischen Wissenschaften, Springer-Verlag, Berlin, 1976.

[4] A. Bespalov, Orthogonal systems of singular functions and numerical treatment of problems with degeneration of data, Adv. Comput. Math., 19 (2003), pp. 159-182. MR1973463 (2004f:65179)

[5] A. Y. Bespalov And V. A. Rukavishnikov, The use of singular functions in the h-p version of the finite element method for the Dirichlet problem with degeneration of the input data, Siberian Journal of Numerical Mathematics, 4 (2001), pp. 201-228.

[6] P. G. Ciarlet, The Finite Element Method for Elliptic Problems, North-Holland, Amsterdam, 1978. MR0520174 (58:25001)

[7] K. ERIKsson, Finite element methods of optimal order for problems with singular data, Math. Comp., 44 (1985), pp. 345-360. MR0777267 (86j:65155a)

[8] — Improved accuracy by adapted mesh-refinements in the finite element method, Math. Comp., 44 (1985), pp. 321-343. MR0777268 (86j:65155b)

[9] K. ERIKSSOn AND V. ThoméE, Galerkin methods for singular boundary value problems in one space dimension, Math. Comp., 42 (1984), pp. 345-367. MR0736441 (85i:65107)

[10] P. Grisvard, Elliptic Problems in Nonsmooth Domains, Pitman Publishing, Inc., Boston, 1985. MR.0775683 (86m:35044)

[11] B. Q. GUO AND I. BABUŠKA, The h-p version of the finite element method, Part 1: The basic approximation results, Comp. Mech., 1 (1986), pp. 21-41.

[12] G. H. Hardy, J. E. Littlewood, and G. Pólya, Inequalities, Cambridge University Press, 1952. MR0046395(13,727e)

[13] D. JeSPERSEN, Ritz-Galerkin methods for singular boundary value problems, SIAM J. Numer. Anal., 15 (1978), pp. 813-834. MR0488786 (58:8296)

[14] L. D. Landau And E. M. Lifshitz, Quantum Mechanics: Non-Relativistic Theory, Pergamon Press, Oxford, 1965.

[15] D. Marini And P. Pietra, Finite element approximation of a degenerate eigenvalue problem, in Boundary Value Problems for Partial Differential Equations and Applications, C. Baiocchi and J. L. Lions, eds., Paris, 1993, Masson, pp. 393-398. MR1260468 (94k:65163)

[16] — Mixed finite element approximation of a degenerate elliptic problem, Numer. Math., 71 (1995), pp. 225-236. MR1347165 (96f:65155)

[17] V. A. Rukavishnikov, On differentiability properties of an $R_{\nu}$-generalized solution of the Dirichlet problem, Dokl. Acad. Nauk SSSR, 309 (1989), pp. 1318-1320. English transl. in: Soviet Math. Dokl., 40 (1990), pp. 653-655. MR.1045325 (91e:35106)

[18] V. A. Rukavishnikov and H. I. Rukavishnikova, The finite element method for the first boundary value problem with compatible degeneracy of the initial data, Dokl. Russ. Acad. Nauk, 338 (1994), pp. 731-733. English transl. in: Russian Acad. Sci. Dokl. Math., 50 (1995), pp. 335-339. MR.1311312 (96c:65183) 
[19] R. Sсотт, Finite element convergence for singular data, Numer. Math., 21 (1973), pp. 317327. MR0337032 (49:1805)

[20] W. TöRNIG, Numerische Mathematik für Ingenieure und Physiker. Band 2: Eigenwertprobleme und Numerische Methoden der Analysis, Springer-Verlag, Berlin, 1979. MR0628143 (84c:65003)

Departamento de Ingeniería Matemática, Universidad de Concepción, Casilla 160-C, Concepción, Chile

E-mail address: darroyo@ing-mat.udec.cl

Computational Center, Far-Eastern Branch of the Russian Academy of Sciences, Khabarovsk, Russia

E-mail address: albespalov@yahoo.com

Bicom, Department of Mathematical Sciences, Brunel University, Uxbridge UB8 3PH, United Kingdom

E-mail address: norbert.heuer@brunel.ac.uk 